\title{
SNX27 and SORLA Interact to Reduce Amyloidogenic Subcellular Distribution and Processing of Amyloid Precursor Protein
}

\author{
Timothy Y. Huang, ${ }^{1}$ Yingjun Zhao, ${ }^{1}$ Xiaoguang Li, ${ }^{1,3}$ Xin Wang, ${ }^{1}$ I-Chu Tseng, ${ }^{1}$ Robert Thompson, ${ }^{1}$ Shichun Tu, ${ }^{1}$ \\ Thomas E. Willnow, ${ }^{2}$ Yun-wu Zhang, ${ }^{1}$ and Huaxi $\mathrm{Xu}^{1}$ \\ ${ }^{1}$ Neuroscience and Aging Research Center, Sanford-Burnham Prebys Medical Discovery Institute, La Jolla, California 92037, ${ }^{2}$ Max Delbrueck Center for \\ Molecular Medicine, 13125 Berlin, Germany, and ${ }^{3}$ Institute of Clinical Pharmacology, Guangzhou University of Chinese Medicine, Guangzhou 510405, \\ China
}

Proteolytic generation of amyloidogenic amyloid $\beta(\mathrm{A} \beta)$ fragments from the amyloid precursor protein (APP) significantly contributes to Alzheimer's disease (AD). Although amyloidogenic APP proteolysis can be affected by trafficking through genetically associated AD components such as SORLA, how SORLA functionally interacts with other trafficking components is yet unclear. Here, we report that SNX27, an endosomal trafficking/recycling factor and a negative regulator of the $\gamma$-secretase complex, binds to the SORLA cytosolic tail to form a ternary complex with APP. SNX27 enhances cell surface SORLA and APP levels in human cell lines and mouse primary neurons, and depletion of SNX27 or SORLA reduces APP endosome-to-cell surface recycling kinetics. SNX27 overexpression enhances the generation of cell surface APP cleavage products such as soluble alpha-APP C-terminal fragment (CTF $\alpha$ ) in a SORLA-dependent manner. SORLA-mediated A $\beta$ reduction is attenuated by downregulation of SNX27. This indicates that an SNX27/SORLA complex functionally interacts to limit APP distribution to amyloidogenic compartments, forming a non-amyloidogenic shunt to promote APP recycling to the cell surface.

Key words: Alzheimer's disease; APP; endosome recycling; protein trafficking; SNX27; SORLA

Significance Statement

Many genes have been identified as risk factors for Alzheimer's disease (AD), and a large proportion of these genes function to limit production or toxicity of the $\mathrm{AD}$-associated amyloid $\beta(\mathrm{A} \beta)$ peptide. Whether and how these genes precisely operate to limit $\mathrm{AD}$ onset remains an important question. We identify binding and trafficking interactions between two of these factors, SORLA and SNX27, and demonstrate that SNX27 can direct trafficking of SORLA and the A $\beta$ precursor APP to the cell surface to limit the production of $A \beta$. Diversion APP to the cell surface through modulation of this molecular complex may represent a complimentary strategy for future development in $\mathrm{AD}$ treatment.

\section{Introduction}

Alzheimer's disease (AD) is a prominent neuropathological disorder linked to the appearance of extracellular amyloid $\beta(\mathrm{A} \beta)$

Received Jan. 19, 2016; revised April 27, 2016; accepted June 9, 2016.

Author contributions: T.Y.H. and H.X. designed research; T.Y.H., Y.Z., X.L., X.W., I.C.T., and R.T. performed research; T.Y.H. and T.E.W. contributed unpublished reagents/analytic tools; T.Y.H., S.T., and Y.-w.Z. analyzed data; T.H.,S.T., Y.-w.Z., and H.X. wrote the paper.

This work was supported in part by National Institutes of Health Grants R01AG021173, R01AG038710, R01AG044420, and R01NS046673 (H.X.) and R21AG049247 (Y.-w.Z.), the Alzheimer's Association (C4C-15-369446 and DSADIIP-13283543 to H.X. and (4C-15-368951 to Y.-w.Z.), and Shiley-Marcos Alzheimer's Disease Research Center University of California San Diego Grant ADRC 201559226 (T.Y.H.). We thank Dr. Wanjin Hong for sorting nexin constructs and for the mouse SNX27 antibody and Dr. Paul Slesinger for antibodies recognizing human SNX27. We also thank members of the Xu laboratory for constructive discussion and Lili Lacarra for animal support.

The authors declare no competing financial interests.

Correspondence should be addressed to Dr. Huaxi Xu, Neurodegenerative Disease Research Program, Sanford Burnham Prebys Medical Discovery Institute, 10901 North Torrey Pines Road, La Jolla, CA 92037. E-mail: xuh@sbpdiscovery.org. peptide aggregates, in which soluble $\mathrm{A} \beta$ multimers enact neurotoxic effects during neurodegenerative onset ( $\mathrm{Tu}$ et al., 2014). Although familial $\mathrm{AD}$ cases are rare, the prevalence of $\mathrm{AD}$ associated familially linked alleles found in the $\mathrm{A} \beta$-derived amyloid precursor protein (APP) and presenilin (PS)1/PS2 $\gamma$-secretase APP cleavage components further demonstrates the importance of $\mathrm{A} \beta$ and $\mathrm{APP}$ processing in $\mathrm{AD}$ neuropathology (Zhang et al., 2011).

APP is a single-pass transmembrane component that shuttles between Golgi, cell surface, and endosomal/lysosomal compartments (Thinakaran and Koo, 2008; Jiang et al., 2014; Wang et al., 2014a). The route by which APP is trafficked determines whether 
APP is proteolytically processed into neurotoxic $A \beta$ peptides through the amyloidogenic pathway or nontoxic APP products such as soluble $\operatorname{APP} \alpha(\operatorname{sAP} \alpha)$ and CTF $\alpha$ (alpha-APP C-terminal fragment) through the non-amyloidogenic pathway. Amyloidogenic and non-amyloidogenic processing is mutually exclusive; APP cleavage by non-amyloidogenic $\alpha$-secretases precludes the availability of the rate-limiting $\beta$-secretase (BACE1) amyloidogenic cleavage site. Interestingly, non-amyloidogenic cleavage of APP by $\alpha$-secretases occurs primarily at the cell surface, whereas amyloidogenic cleavage by BACE1 and the $\gamma$-secretase complex requires acidified trans-Golgi and endosomal environments (Xu et al., 1997; Jiang et al., 2014). This suggests that intracellular APP trafficking components also likely influence amyloidogenic APP processing.

In support of this notion, APP trafficking components such as SORLA (sortilin-related receptor with A-type repeats) have been found to be linked to AD (Pottier et al., 2012). Originally identified as a component reduced in AD brain (Scherzer et al., 2004), SORLA deletion has been shown to aggravate $\mathrm{A} \beta$ plaque formation in AD mouse models (Andersen et al., 2005; Rohe et al., 2008), whereas murine SORLA overexpression was shown to reverse $\mathrm{A} \beta$ generation (Caglayan et al., 2014). As an APP trafficking component, SORLA may act as a retention factor to sequester APP at the Golgi away from amyloidogenic processing at the endosome (Andersen et al., 2005; Rogaeva et al., 2007; Fjorback et al., 2012). However, the presence of SORLA at the cell surface and endosomal compartments (Andersen et al., 2005; Offe et al., 2006; Herskowitz et al., 2012) suggests that SORLA may likely have additional roles in mediating endosome-to-cell surface trafficking. Indeed, if mechanisms for SORLA-mediated APP endosome-to-cell surface recycling could be enhanced and restored in SORLA-attenuated $\mathrm{AD}$ patients, this may attenuate $\mathrm{A} \beta$ neurotoxicity associated with age onset.

SNX27 is a trafficking component required in driving endosome-to-cell surface transport of various endosomal targets through cargo-selective interactions with its N-terminal PDZ domain. Proteomic analysis of cell surface components with siRNAmediated SNX27 depletion suggests that SNX27 may be required for APP trafficking, possibly through an intermediary trafficking component, because no binding interactions were detected between APP and SNX27 in vivo (Steinberg et al., 2013). Although a role for $\mathrm{SNX} 27$ in reducing amyloidogenic $\mathrm{A} \beta$ generation through interactions with PS1/ $\gamma$-secretase has also been implicated (Wang et al., 2014b), whether and how SNX27 can exert cytoprotective effects through its ability to influence APP trafficking remains elusive.

Here, we describe a mechanism for SORLA endosome-toplasma membrane recycling, which concurrently results in increased surface APP distribution and concomitant nonamyloidogenic $\alpha$-secretase cleavage. Through an interaction screen to detect binding interactions between the cytosolic SORLA tail region and retromer complex components, we observe strong interactions between the SNX27 PDZ domain and the SORLA tail. We find that overexpression of SNX27 can enhance surface distribution of both SORLA and APP in cultured cells and neurons, whereas SNX27 depletion in cell lines and haploinsufficiency in primary neurons reduces cell surface SORLA and APP levels. SNX27 overexpression was also found to elevate $\operatorname{sAPP} \alpha$ generation in cultured cells. Likewise, SORLA overexpression in cultured cells was found to attenuate $A \beta$ levels in a SNX27-dependent manner. Together, these results indicate that SNX27 and SORLA interact and provide an endosomal shunt mechanism to shift the endosomal APP trafficking milieu in favor of non-amyloidogenic processing at the cell surface.

\section{Materials and Methods}

Cell culture and transfection. HEK293T and HEK293 cells stably expressing the Swedish APP KM670/671NL variant (HEKswAPP) were cultured in DMEM supplemented with 10\% FBS. Turbofect transfection reagent (Life Technologies) was used for transient transfection of all cell lines described according to specifications from the manufacture supplier. RNAi MAX (Life Technologies) was used for transfection of siRNA oligonucleotides. siRNA targeting sequences to cognate human targets for cell line transfection were $5^{\prime}$-taccagatggaacaacggtta for SNX27 and 5' ctgggatttatcggagcaata for SORLA, all transfected at a final concentration of $10 \mathrm{~nm}$ and purchased from Qiagen. An AllStars siRNA oligo was transfected as a negative control (Qiagen).

Primary neuronal culture. Pregnant female mice were collected from timed matings, and embryos were harvested from $S N X 27^{+/-}$het/wildtype (WT) matings at E15-E17. Primary cortical neurons were obtained by microdissection of the cerebral cortex from embryos using a stereomicroscope and dispersed by digestion in trypsin and DNAseI for $30 \mathrm{~min}$ at $37^{\circ} \mathrm{C}$, followed by trituration in DMEM. Embryonic tissue was also collected at harvesting and processed for genotype analysis using the MyTaq DNA extraction and PCR genotyping system (Bioline), in which WT and $S N X 27^{+/-}$het neurons from individual embryos were maintained separately. Neurons were plated and maintained on poly-D-lysinecoated coverslips or culture dishes in Neurobasal medium supplemented with B27, glutamine, and penicillin/streptomycin, whereby media was changed every $3 \mathrm{~d}$, when half of the media was replaced.

Antibodies and plasmid constructs. GST-fusion constructs were expressed in HEK293T cells using the pRK5mGST vector, in which fulllength SNX27 and various domain deletions were cloned downstream in-frame of the N-terminal GST tag. The SORLA cytosolic tail (amino acids 2159-2214) region and deletions (tail $\Delta 2159-2177$ comprising amino acids 2160-2214, tail-2182, amino acids 2159-2182, tail-2198, and amino acids 2159-2198), VPS26, VPS29, and VPS35 were also cloned into pRK5mGST for expression in HEK293T cells for interaction studies. Full-length pRK5mGST SNX27 H114A was generated by PCR-mediated site-directed mutagenesis, using a pRK5mGST SNX27 template. Myctagged retromer expression vectors were generous gifts from Dr. Wanjin Hong (Institute of Molecular and Cell Biology, Singapore): pDmycVPS26 and VPS35, and pCIneo myc-SNX27, SNX1, SNX2, SNX5, and SNX6. pCDNA3 SORLA was obtained as a kind gift from Dr. James Lah (Emory University, Atlanta, GA). Vectors to express recombinant GST and His6 proteins included pGEX4T3-SORLA tail in which SORLA cDNA sequences corresponding to amino acids 2159-2214 were PCR amplified and cloned using BamHI/EcoRI sites; likewise, pTRChis6A SNX27, pTRChis6A SNX27 PDZ, and pTRChis6A VPS26 were PCR amplified and cloned in-frame downstream of the his 6 tag. Coexpression of SNX27 and GFP was mediated by transfection of pIRES2 GFP-SNX27, in which SNX27 was cloned using BamHI/XhoI sites into BglII/SalI vector sites. Lentiviruses for SNX27 transduction were generated by the SBPMDI viral vector core facility using a pCDH513 SNX27 vector, in which SNX27 was cloned into pCDH513 using NheI/BamHI sites.

pRK5mGST-SNX27 PDZ constructs were generated by sequence alignment of SNX27, NHERF2, and SHANK2 PDZ domains, in which conserved amino acids within the secondary $\beta$-strand and $\mathrm{H} 114 \mathrm{~A}$ $\alpha$-helix residues were mutated by site-directed mutagenesis. Because all residues conferred charged or bulky structural elements except for the terminal L130 residue within the terminal $\beta$-strand, we switched the hydrophobic $\mathrm{L} 130$ residue to $\mathrm{Q}$.

pCDNA3 SORLA-mCherry was cloned by generating an XbaI at the $3^{\prime}$ end to replace the SORLA stop codon, in which mCherry was cloned downstream of the SORLA ORF using XbaI sites. A pCDNA3 GFPSNX27 expression construct used to detect overlapping SNX27/SORLA localizing interactions in HEK293T cells was generated by cloning the SNX27 ORF downstream of an N-terminal GFP tag. GFP-tagged GLUR1 and SORLA cytosolic tail constructs were cloned into pCDNA3 GFP with a six glycine linker, in which cDNA sequences corresponding to the GLUR1 (amino acids 831-906) and SORLA (amino acids 2159-2214) tail 
sequences were cloned in-frame downstream. A pCDNA3-FLAG SNX27 vector construct was generated by cloning SNX27 in-frame downstream of pCDNA3-FLAG.

Antibodies detecting human and mouse SNX27 were generous gifts from Drs. Wanjin Hong and Paul Slesinger (Mount Sinai Hospital, New York, NY). SORLA (LR11) and EEA1 (BD Biosciences), BIP, $\gamma$-adaptin, and GST polyclonal (Santa Cruz Biotechnology), FLAG M2 (Stratagene), GFP monoclonal (Genetex), TGN38 and Y188 C-terminal APP rabbit monoclonal (Abcam), actin (Sigma), Xpress (Life Technologies), Lamp1 (Ly1C6; Enzo Diagnostics), and TnfR (Life Technologies) antibodies were all purchased from commercial sources. The 9E10 monoclonal antibody to detect the myc epitope was generated in-house. The B436 monoclonal antibody targeting the human extracellular $A \beta$ transmembrane proximal region has been described previously (Eggert et al., 2009) and was used to detect $\mathrm{A} \beta, \operatorname{sAPP} \alpha$, and surface APP by immunofluorescence. The 22C11 monoclonal antibody for APP detection was purified in-house.

Recombinant protein purification. GST, GST-SORLA tail, and his6SNX27, SNX27 PDZ, and VPS26 were expressed from pGEX4T3 and pTRChis6 A vectors described above and purified by glutathione Sepharose/Ni-NTA agarose affinity purification methods described previously (Huang et al., 2013). BL21 pGEX4T3 or pTRChis6 transformants were grown to exponential phase and induced with $1 \mathrm{~mm}$ isopropyl- $\beta$ D-thiogalactopyranoside and cultured for an additional 3-5 h. Bacteria were pelleted in lysis buffer and lysed by sonication in $10 \mathrm{mg} / \mathrm{ml} \mathrm{lysozyme}$ in the presence of protease inhibitors (complete; Roche). After clearing lysates by spinning at $10,000 \times g$ for $10 \mathrm{~min}$, GST proteins were precipitated using glutathione Sepharose, whereas his6-tagged constructs were precipitated with Ni-NTA agarose in the presence of $10 \mathrm{~mm}$ imidazole.

Glutathione beads were washed in $10 \mathrm{~mm}$ Tris- $\mathrm{HCl}, \mathrm{pH} 8$, and $0.5 \mathrm{M}$ $\mathrm{NaCl}$, whereas Ni-NTA beads were washed in the same buffer containing $20 \mathrm{~mm}$ imidazole. GST proteins were eluted with $30 \mathrm{~mm}$ reduced glutathione in $0.3 \mathrm{M}$ Tris- $\mathrm{HCl}$, and his 6 proteins were eluted in $0.3 \mathrm{M}$ imidazole in $10 \mathrm{~mm}$ Tris- $\mathrm{HCl}, \mathrm{pH} 8$, and $0.5 \mathrm{~m} \mathrm{NaCl}$. Eluted proteins were then dialyzed in $1 \times$ PBS with $5 \%$ glycerol and $0.3 \mathrm{~mm}$ DTT overnight and frozen at $-80^{\circ} \mathrm{C}$. The Xpress antibody was used to detect the Xpress epitope downstream of the his6 tag in the pTRChis6A vector by immunoblot.

Recombinant in vitro, semi-in vitro, and in vivo GST pull-down assays. To assay binding interactions between recombinant GSTSORLA tail and his6 purified SNX27/VPS26 constructs, recombinant purified GST or GST-SORLA tail were incubated with recombinant his6 proteins for $2 \mathrm{~h}$ at $4^{\circ} \mathrm{C}$ rocking in the presence of glutathione Sepharose, precipitated, and washed three times at room temperature $15 \mathrm{~min}$ each in lysis buffer containing $0.5 \mathrm{~m} \mathrm{NaCl}$. GST and his6 components were then immunoblotted for GST or Xpress bound/ coprecipitated by immunoblotting.

Semi-in vitro binding interactions required reimmobilizing recombinant purified GST or GST-SORLA tail constructs on glutathione, in which beads were washed and individually incubated with HEK293T lysates expressing myc-tagged constructs comprising the core retromer complex (Vps26, Vps35, Snx27, Snx1, Snx2, Snx5, and Snx6). After incubation with the immobilized GST constructs, myc-tagged constructs were coprecipitated, washed in lysis buffer, and visualized by immunoblotting.

In vivo GST pull-down interactions involved transfection of HEK293T cells with pRK5mGST vectors expressing retromer components or GSTtagged SNX27 truncation fragments as described above. For detection of semi-endogenous interactions, pRK5mGST vectors expressing SNX27, SNX27 truncations, or other tagged retromer components were transfected in HEK293T and cultured overnight, and lysates were generated, in which GST constructs were precipitated using glutathione agarose. Beads were consequently washed, and endogenous SORLA or APP interactions coprecipitated with the GST precipitates were detected by immunoblotting. In our PDZ mutagenesis screen for mutations attenuating SNX27 PDZ/SORLA interactions, pRK5mGST PDZ constructs were transfected in HEKswAPP cells and precipitated for endogenous SORLA or APP with glutathione Sepharose as above. We note in our pull-down assays that L130Q and L130A mutations have equivalent effects on attenuating SORLA/APP interaction (data not shown).

Coimmunoprecipitation assays. SORLA complexes were immunoprecipitated from mouse brain lysates in lysis buffer using $2 \mu \mathrm{g}$ of SORLA monoclonal antibody (BD Biosciences), and immune complexes were precipitated with Protein $G$ and washed five times in lysis buffer before immunoblotting.

Cell surface biotinylation. Cell surface biotinylation using EZ-link Sulfo-NHS-LC biotin in HEKswAPP and primary neurons was performed as described previously (Wang et al., 2013). Briefly, cells or neurons were washed in cold $1 \times$ PBS supplemented with $1 \mathrm{mM} \mathrm{MgCl}_{2}$ and $1.3 \mathrm{mM} \mathrm{CaCl}_{2}(1 \times \mathrm{PBS} / \mathrm{CM})$ and incubated with $500 \mu \mathrm{g} / \mathrm{ml}$ biotin labeling reagent in $1 \times \mathrm{PBS} / \mathrm{CM}$ at $4^{\circ} \mathrm{C}$ with agitation. Labeling was repeated and quenched with $7.5 \mu \mathrm{g} / \mathrm{ml}$ glycine in $1 \times$ PBS/CM. Cells were washed, lysed in lysis buffer, and incubated overnight with streptavidin agarose at $4^{\circ} \mathrm{C}$ with agitation. Streptavidin agarose beads were then washed and boiled in Laemmli buffer, and biotin-labeled precipitates were visualized and quantified by immunoblotting.

Kinetic endocytosis and endosome to surface recycling assays. Measurement of cell surface internalization and endosome-to-cell surface recycling of SORLA and APP was determined in transfected HEKswAPP cells as described previously (Wang et al., 2013).

Sucrose density fractionation. For sucrose density subcellular fractionation, cells were lysed using a steel ball-bearing chamber and subjected to ultracentrifugation as described previously (Zhang et al., 2005). Briefly, cells were washed in $1 \times$ PBS and resuspended in $0.25 \mathrm{M}$ sucrose, $10 \mathrm{~mm}$ Tris- $\mathrm{HCl}, \mathrm{pH} 7.4$, and $1 \mathrm{~mm} \mathrm{MgAc}_{2}$ and passed 15-20 times in a steel ball-bearing chamber. Lysates were cleared at $800 \times g$ for $5 \mathrm{~min}$ and loaded onto a discontinuous sucrose gradient comprising $1.5 \mathrm{ml}$ of $2 \mathrm{M}, 4$ $\mathrm{ml}$ of $1.3 \mathrm{M}, 3 \mathrm{ml}$ of $1.16 \mathrm{M}$, and $2 \mathrm{ml}$ of $0.8 \mathrm{M}$ sucrose in $10 \mathrm{~mm}$ Tris- $\mathrm{HCl}$, $\mathrm{pH} 7.4$, and $1 \mathrm{~mm} \mathrm{MgAc}_{2}$. Gradients were spun for $2.5 \mathrm{~h}$ at $100,000 \times g$, and $1 \mathrm{ml}$ fractions were collected and subjected to TCA precipitation overnight at $4^{\circ} \mathrm{C}$, washed in acetone, and subjected to immunoblot analysis.

Mouse lines and hippocampal fractionation analysis. TG2576 mouse lines expressing the Swedish APP695 KM670/671NL allele were purchased from Taconic and maintained in-house. Mouse lines overexpressing SORLA at the Rosa26 locus were described previously (Caglayan et al., 2014); SORLARosa26 and SNX27 $7^{+-}$lines were crossed with TG2576 transgenics (TGs) to generate SORLA-Rosa26/TG2576 and SNX27 $7^{+/} /$TG2576 lines. Triple TG2576/SORLA-Rosa26/SNX27 $7^{+/-}$TG mouse lines were generated by crossing TG2576/SORLA-Rosa26-positive animals with $S N X 27^{+/-}$or TG2576/SNX27 ${ }^{+/-}$animals with SORLA-Rosa26 TGs. Genotyped TG2576 TGs from these triple crosses were selected for analysis, in which SNX27 WT and $S N X 27^{+/-}$animals were compared in SORLA-Rosa26 WT and TG (overexpression) backgrounds.

TG2576 mice from these triple TG crosses were killed at 8 weeks of age, whereby the hippocampus was dissected and frozen at $-80^{\circ} \mathrm{C}$. Hippocampal tissue was processed for biochemical synaptic/PSDenrichment analysis as described previously (Wang et al., 2013). Briefly, hippocampal tissue was homogenized using a Dounce homogenizer in fractionation buffer ( $0.32 \mathrm{~m}$ sucrose and $25 \mathrm{~mm}$ HEPES, $\mathrm{pH} 7.4$ in protease and phosphatase inhibitors), and nuclei and debris was cleared by centrifugation $(500 \times g, 10 \mathrm{~min})$. Membranes were separated by centrifugation at $10,000 \times \mathrm{g}$ for $12 \mathrm{~min}$, and membranes were washed twice in $25 \mathrm{~mm}$ HEPES, pH 7.4, and $150 \mathrm{~mm} \mathrm{NaCl}$ and solubilized in $25 \mathrm{~mm}$ HEPES, pH 7.4, and $150 \mathrm{~mm} \mathrm{NaCl}$ buffer with $1 \%$ Triton X-100 with phosphatase/protease inhibitors. PSD-enriched fractions were precipitated by centrifugation at $10,000 \times g$ for $20 \mathrm{~min}$, washed in $25 \mathrm{~mm}$ HEPES, pH 7.4, and $150 \mathrm{~mm} \mathrm{NaCl}$, and solubilized in $25 \mathrm{~mm}$ HEPES, $\mathrm{pH}$ $7.4,150 \mathrm{~mm} \mathrm{NaCl}$, and $1 \%$ Triton X-100. A total of six mice $(n=6)$ for each genotype $(n=24$, total) were dissected, and hippocampal tissues were processed for fractionation.

All procedures involving animals were performed under the guidelines of Xiamen University and Sanford-Burnham Medical Research Institute Institutional Animal Care and Use Committee.

Microscope image sample preparation and acquisition. All images were acquired using an inverted Zeiss Axio Observer Z1 fluorescence microscopy system, in which acquisition and processing of images used Slide- 
book 5.5 software. Cultured HEKswAPP and primary neurons described above were seeded and manipulated on poly-D-lysine-coated glass coverslips and fixed in $4 \%$ paraformaldehyde (PFA). After permeabilization in $0.5 \%$ Triton X-100 in $1 \times$ PBS, coverslips were washed and blocked in $3 \%$ BSA and subsequently stained in primary and fluorescent secondary antibodies before mounting in Prolong Gold antifade on glass slides.

For cell surface detection of APP in HEKswAPP cells, cells were allowed to cool to $4^{\circ} \mathrm{C}$ in cold $1 \times$ DMEM and labeled with B436 antibody for $15 \mathrm{~min}$ at $4^{\circ} \mathrm{C}$. Cells were then washed with cold PBS and fixed in $4 \%$ PFA, followed by blocking, fluorescent secondary antibody labeling, and mounting. APP fluorescence images by surface labeling using the B436 antibody were acquired using matched exposure settings, and threshold levels for fluorescence channels were adjusted equivalently. The APP fluorescence channel was extracted, and fluorescence intensity of the acquired images were measured using NIH ImageJ.

Statistical analyses. All statistical analyses used in this study determined the significant differences between two groups using nonpaired $t$ tests with equal variance from a minimum of three experiments unless stated differently.

\section{Results}

\section{SNX27 interacts with the SORLA cytosolic tail}

The retromer complex has been previously described to selectively bind and direct cargo from endosomal compartments. Because trafficking mechanisms for SORLA selection and subsequent distribution from the endosome has yet to be described, we determined whether individual components of the retromer complex could interact with the SORLA cytosolic tail region. Using a semi-in vitro interaction assay with a recombinant purified GST-SORLA tail fusion peptide immobilized on glutathione beads, we find that SNX27 selectively precipitated with the SORLA cytosolic tail with little interaction observed from other components of the retromer complex (Fig. 1A).

To confirm these interactions, we expressed GST fusions with members of the core retromer components in HEK293T cells and assayed for coprecipitation with endogenous SORLA. We similarly observed selective interactions between SNX27 and endogenous SORLA, with relatively no interaction observed with other retromer components (Fig. 1B). Because SNX27 interactions with endogenous SORLA appeared to be somewhat weak, we also assayed for SNX27/SORLA interactions with SORLA cooverexpression (Fig. 1C). We found that SORLA/SNX27 interactions were indeed markedly improved with SORLA/GST-SNX27 co-overexpression and subsequent precipitation of GST-SNX27 complexes (Fig. 1C). Because SNX27 has been previously characterized to bind various endosomal trafficking components through selective interactions with modular PDZ, PX, RA, and FERM domains (Cullen, 2008), we expressed individual GSTfused SNX27 domain modules in HEK293T cells and assayed for interactions with endogenous SORLA (Fig. 1D). We observed strong interactions with full-length SNX27 and the N-terminal SNX27 PDZ domain with endogenous SORLA (Fig. 1D), in which relatively little interaction was observed in constructs lacking this domain. Using recombinant purified components, we next determined whether interactions between the SORLA tail and SNX27 were direct. We found that full-length SNX27 or the SNX27 PDZ domain coprecipitated with the SORLA cytosolic tail (Fig. 1E). These results suggest that SNX27/SORLA tail interactions may be direct. As interactions between SORLA and VPS26 were previously described (Fjorback et al., 2012), we also could reiterate VPS26/SORLA tail interactions in vivo (Fig. $1 F$ ) but not in vitro (Fig. 1E), suggesting that VPS26 may require complexes formed in vivo for SORLA tail interactions.

SORLA interactions with VPS26 were previously reported to require a FANSHY motif within amino acids $2159-2180$ within the SORLA tail (Fjorback et al., 2012); we find by deletion and subsequent GST pull-down analysis that deletion of the membrane proximal 2159-2177 region of the SORLA tail attenuated its interaction with both VPS26 and SNX27 in cells coexpressing GST-SNX27 or GST-VPS26 and GFP-SORLA tail constructs (Fig. $1 F$ ). Because we were able to reconstitute SORLA tail/ SNX27 interactions in vitro using recombinant proteins (Fig. 1E), we determined the effects of deletion of GGA (amino acids 2208 2214), acidic (amino acids 2190-2198), and membrane proximal (amino acids 2159-2177) regions in the SORLA tail region for interactions with endogenous SNX27 (Fig. 1G). Likewise, deletion of this 2159-2177 region in GST-SORLA tail construct abrogated its interaction with endogenous SNX27, whereas deletion of 2182-2214 (acidic and GGA) or 2198-2214 (GGA) regions had no effect on their interactions (Fig. $1 G$ ). Together, this indicates that SNX27/SORLA interaction likely requires the presence of a membrane proximal region within the SORLA tail.

Extensive evidence suggests that the SNX27 PDZ domain comprises an important cargo selection module required for endosomal sorting. Because SORLA lacks a canonical C-terminal $\mathrm{X}-\mathrm{S} / \mathrm{T}-\mathrm{X}-\Phi \mathrm{PDZ}$ interaction motif, we were interested in determining whether PDZ mutations that abrogate PDZ/PDZ-motif interactions could affect SORLA tail interactions (Lauffer et al., 2010). We found that, although the H114A mutation within an $\alpha$-helical PDZ-motif-binding interface of SNX27 disrupted its interactions with a PDZ-motif substrate GLUR1 tail (Wang et al., 2013), this mutation had relatively little effect on SORLA tail interactions (Fig. 1H). Quantification and comparison of GLUR1 and SORLA tail interactions with SNX27 suggest that SORLA tail/SNX27 interactions in vivo are weak compared with GLUR1 tail/SNX27 binding (Fig. 1H, top graph); however, GLUR1 tail interactions with WT SNX27 are fivefold higher compared with $\mathrm{H} 114 \mathrm{~A}$, in which little or no change is observed between WT/H114A SNX27 and SORLA tail (Fig. $1 H$, middle and bottom graphs). Together, these results indicate a direct interaction between a membrane proximal region of the SORLA cytosolic tail and the SNX27 PDZ domain, which may occur through a different binding mechanism previously described for PDZ/ PDZ C-terminal motifs.

SNX27 and SORLA are components that have been previously observed to localize in part to endosomal compartments (Lauffer et al., 2010; Herskowitz et al., 2012). Given their ability to interact physically, we were interested in determining whether SNX27 and SORLA interactions could be reiterated by localization overlap. mCherry-tagged SORLA and GFP-tagged SNX27 appeared to colocalize at punctate subcellular foci as previously described, and, although no specific overlap was observed with GFP alone, we observed an overlap between SORLA and SNX27 puncta in HEK293 cells stably transfected with the Swedish K595N/M596L variant (HEKswAPP) and primary cortical neurons (Fig. 2A,B). Although overexpressed SORLA and SNX27 may not reconstitute true intracellular distribution profiles of these components, colocalizing overlap between SORLA and SNX27 further support binding interactions between these two components.

\section{SNX27 is important for SORLA and APP cell surface distribution}

As a key trafficking component in mediating the intracellular distribution of APP, SORLA has been shown previously to interact with APP. Therefore, we determined whether endogenous SNX27 could interact with APP and SORLA in vivo by coimmunoprecipitation. Using a SORLA antibody, we found that SORLA could coimmunoprecipitate with both APP and SNX27 from 


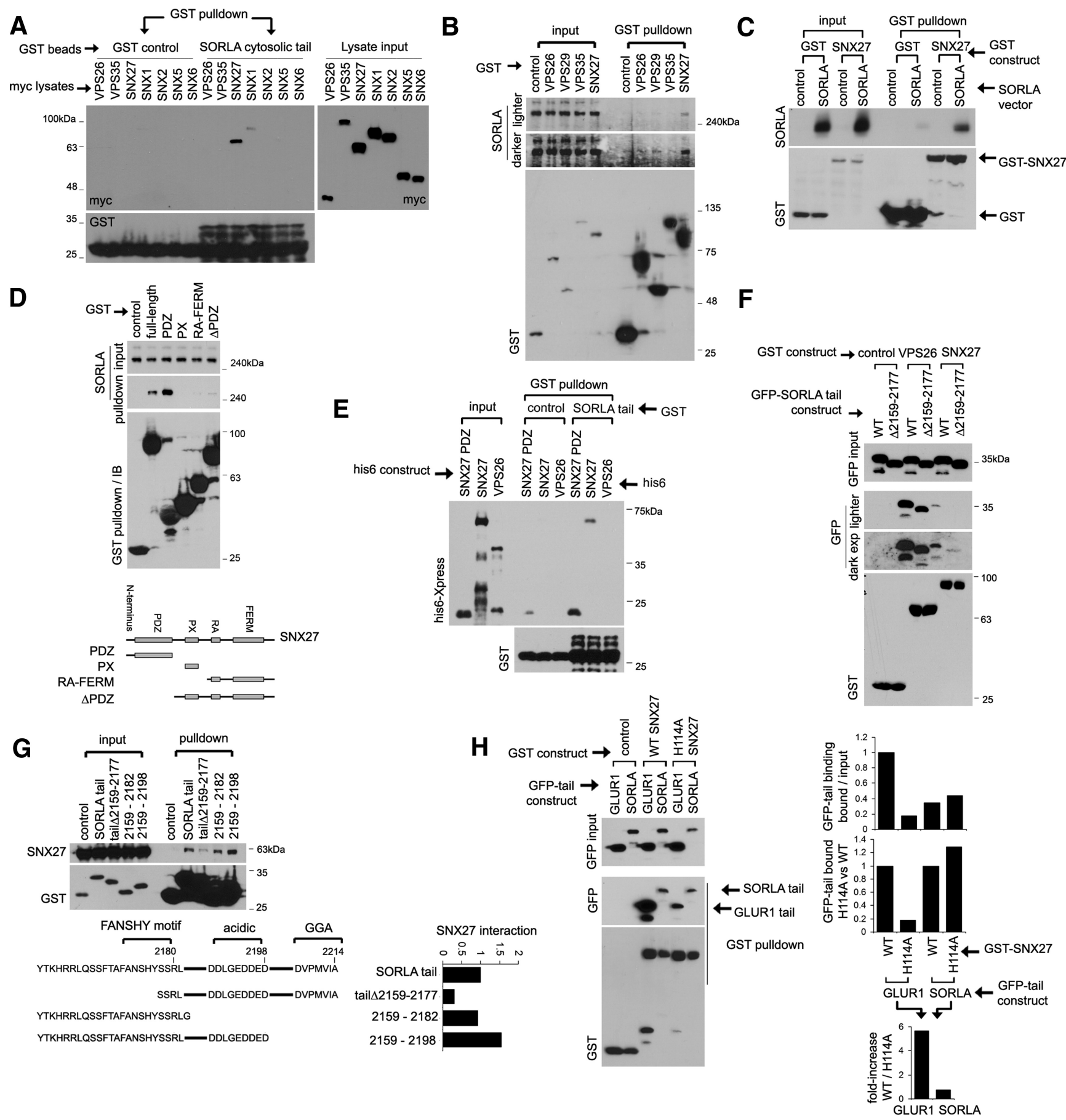

Figure 1. The SNX27 PDZ domain interacts with the cytosolic SORLA tail. A, Probing interactions between the retromer complex and the SORLA cytosolic tail. HEK293T lysates expressing different myc-tagged retromer components were incubated with recombinant purified GST fusions of the SORLA cytosolic tail immobilized on glutathione Sepharose. Unbound components were washed from precipitates, and bound components were detected by myc immunoblots. $B$, The SNX27 retromer component interacts with endogenous SORLA with a comparatively high affinity. GST-fusion constructs comprising various retromer complex components were expressed in HEK293T cells, and glutathione precipitates were probed for endogenous SORLA by immunoblotting. C, GST, GST-SNX27, and control or SORLA vector constructs were coexpressed by transfection in HEKSwAPP cells as indicated, and GST-tagged complexes were precipitated and immunoblotted as in B. D, The SNX27 PDZ domain binds to the SORLA cytosolic tail. GST-SNX27 constructs comprising various SNX27 domains were assayed for coprecipitation with endogenous SORLA in HEK293T cells, in which glutathione precipitates were probed by immunoblotting. E, Reconstitution of SNX27 PDZ domain/SORLA tail interactions in vitro. Recombinant his6-Xpress SNX27, SNX27 PDZ, or VPS26 constructs were incubated with a recombinant purified GST-SORLA tail peptide immobilized to glutathione Sepharose. Bound his6/Xpress-tagged precipitates were detected by Xpress immunoblotting.F, G, SNX27/SORLA tail interactions require residues 2159-2177 in the SORLA tail region. F, GST-tagged VPS26 or SNX27 was coexpressed with a GFP-tagged construct comprising the SORLA tail or SORLA tail lacking a region containing the FANSHY motif (amino acids 2159-2177) in HEK293T, and glutathione precipitates were analyzed by immunoblot. G, GST-tagged SORLA tail deletion constructs were expressed in HEK293T cells, and glutathione precipitates were probed for endogenous SNX27 by immunoblotting. $\boldsymbol{H}$, Vectors expressing GFP-tagged GLUR1 or SORLA cytosolic tail fragments were cotransfected with GST-tagged SNX27 or H114A SNX27 constructs in HEK293T cells, and glutathione Sepharose precipitates from lysates were probed for GFP and GST constructs. Quantification of GFP-tail coprecipitation in the adjacent graphs: bound/input in relation to GLUR1-tail precipitation (GLUR1-tail set to 1.0, top), bound/input of H114A constructs compared with WT for GLUR1 and SORLA tails (WT set to 1.0, middle), and fold increase in GFP-tail binding ratios, WT/H114A for GLUR1 and SORLA tails (bottom). 
A

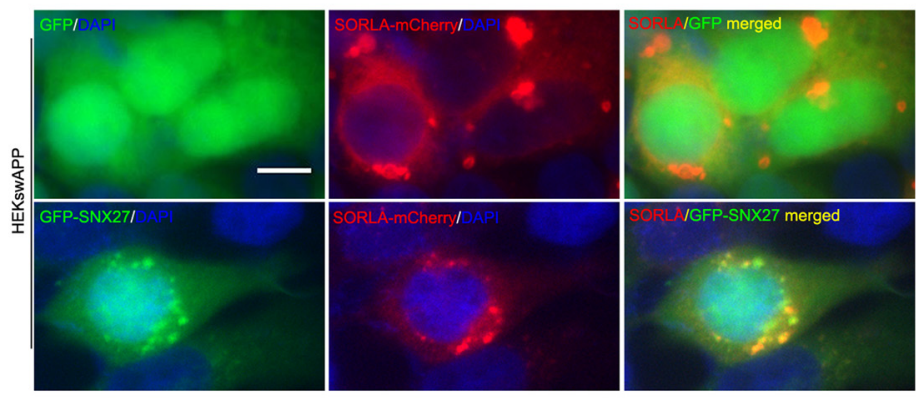

B

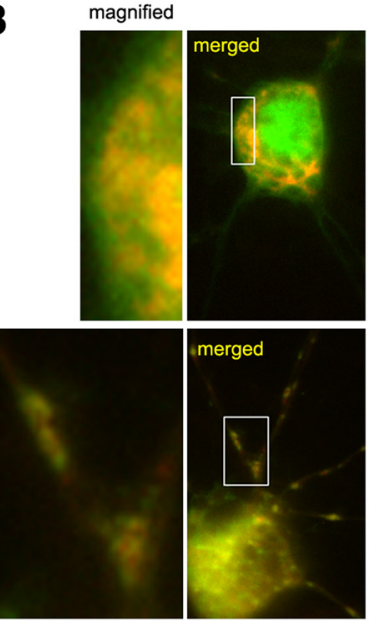

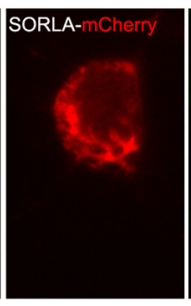
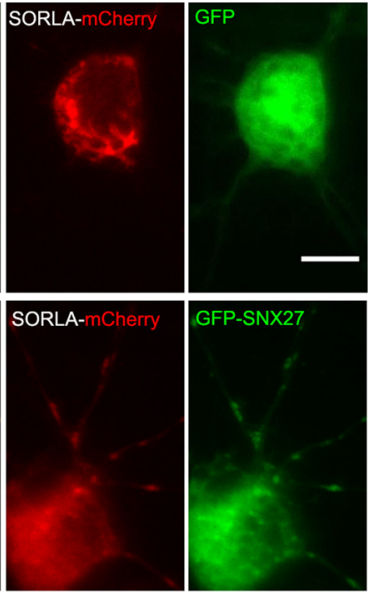

Figure 2. SORLA and SNX27 localization overlap in cells and neurons. A, B, SORLA and SNX27 localization overlap as observed by cotransfection of plasmids expressing either GFP or GFP-SNX27 with SORLA-mCherry in HEKswAPP $(\boldsymbol{A})$ or rat cortical neurons $(\boldsymbol{B})$. Scale bar, $5 \mu \mathrm{m}$.
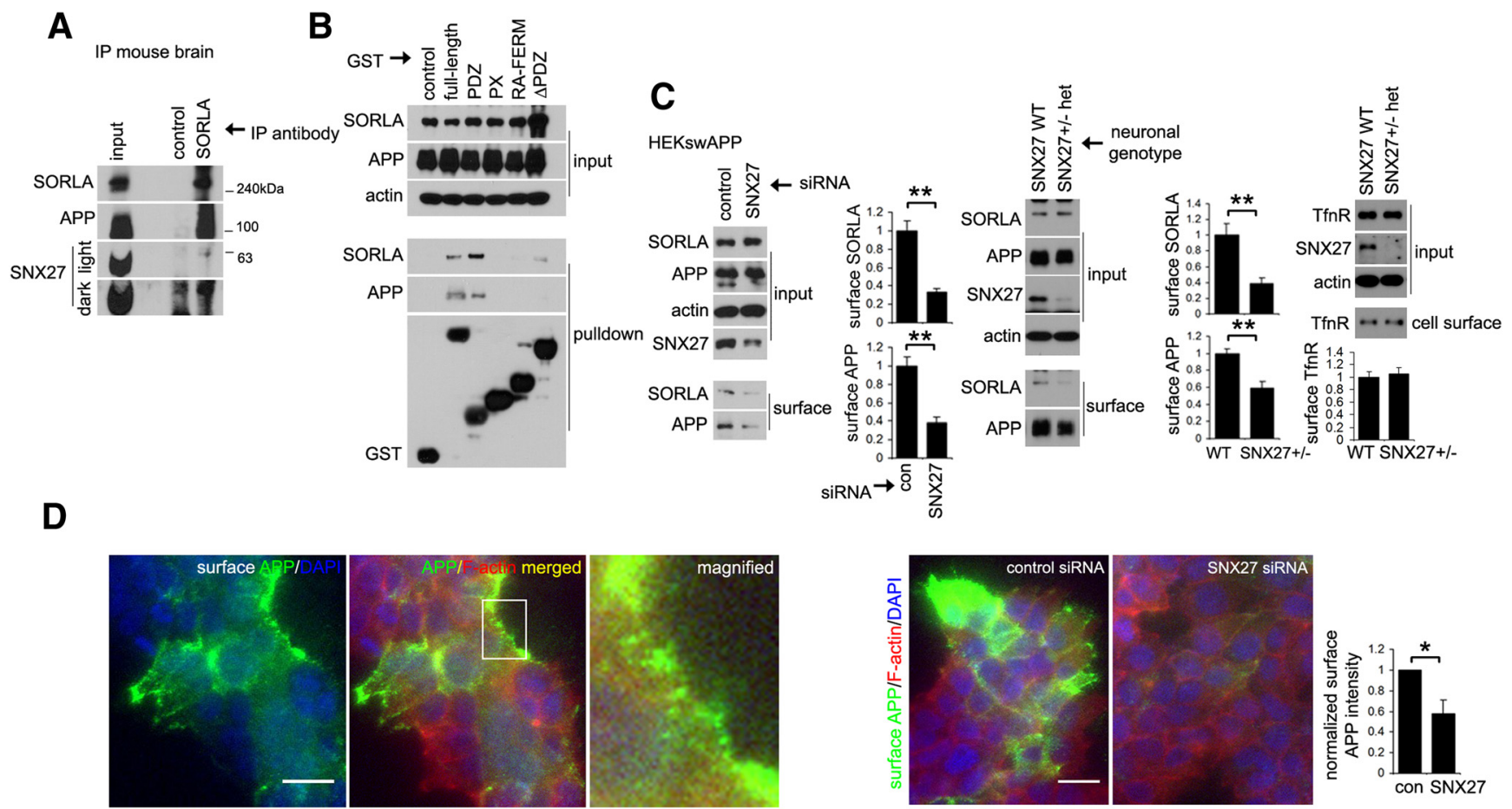

Figure 3. SNX27 interacts with SORLA and APP to maintain surface SORLA/APP levels. A, B, SORLA, APP, and SNX27 form a multimeric complex. A, Precipitation of an endogenous SORLA complex from mouse brain. Cleared mouse brain lysates were incubated with control or SORLA antibodies as indicated, and immune complexes were precipitated with Protein G. Precipitates were then analyzed by immunoblotting. $\boldsymbol{B}$, The SNX27 PDZ domain associates with SORLA and APP. GST-tagged SNX27 deletion constructs were expressed in HEK293T cells, and glutathione Sepharose precipitates were analyzed for endogenous SORLA and APP by immunoblotting. C, SNX27 reduction or haploinsufficiency attenuates surface SORLA/APP levels. HEKswAPP cells were transfected with control or SNX27 siRNA, surface labeled with biotin, precipitated with streptavidin agarose, and immunoblotted for surface SORLA/APP. Primary cortical SNX27 WT or + / - neurons were surface labeled with biotin at DIV7, precipitated with streptavidin agarose, and immunoblotted for SORLA, APP, or TfnR. Values were depicted as mean \pm SE from at least three experiments, where $t$ test equal variance $p$ values were ${ }^{* *} p<0.003$. D, Surface labeling APP (left panels), HEKswAPP cells were surface labeled with the B436 antibody (to recognize APP, green) and costained with phalloidin (to visualize F-actin, red). Effect of SNX27 depletion on surface APP (right panels), HEKswAPP cells transfected with control or SNX27 siRNA were surface labeled with the B436 antibody (green) and costained for $\mathrm{F}$-actin (red). Fluorescence intensity of surface APP under normalized image acquisition conditions were quantified using NIH ImageJ to depict an average normalized value \pm SE from four experiments $\left({ }^{*} p<0.03\right)$. Scale bar, $10 \mu \mathrm{m}$.

mouse brain lysates (Fig. 3A). Similar to SORLA, SNX27 coprecipitates with both endogenous SORLA and APP in a manner dependent on the N-terminal PDZ domain as determined by deletion and GST pull-down analysis (Fig. 3B). These results indicate that SNX27, SORLA, and APP can form a ternary complex.

SNX27 is an endosomal trafficking component that mediates cell surface distribution of numerous transmembrane cargo. We then determined whether reductions in SNX27 levels could influence cell surface SORLA and APP distribution. We found that SNX27 siRNA treatment in HEKswAPP cells led to a reduction in surface SORLA and APP levels (Fig. 3C, left panels). Similarly, we also found that SNX27 haploinsufficiency in dissociated primary neuronal cultures also caused reductions in SORLA and APP cell surface distribution with little influence on surface transferrin 
A

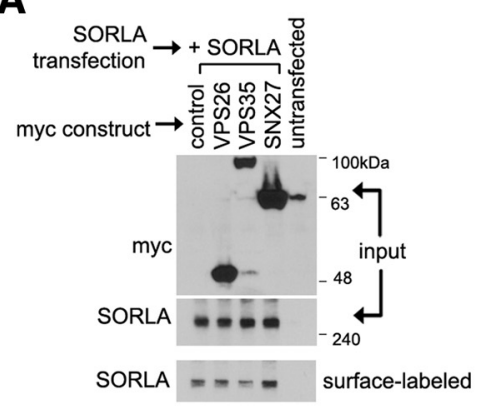

B

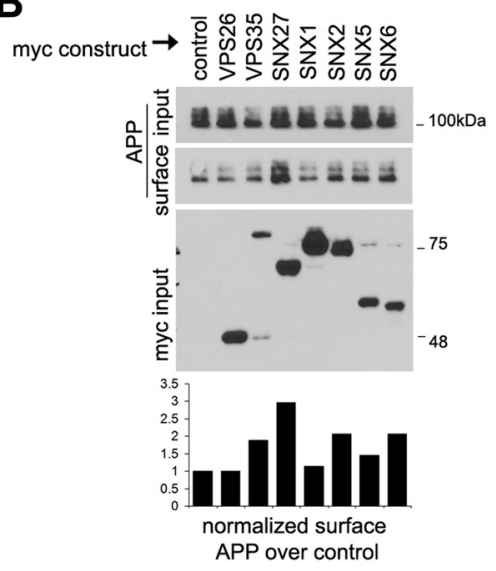

C

HEK293SWAPP

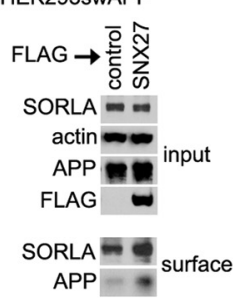

surface

D

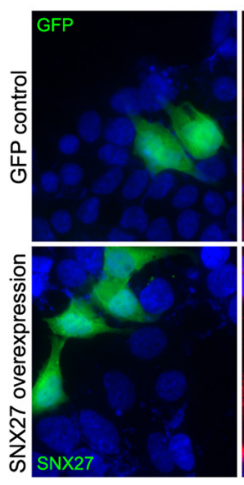

Primary cortical neurons

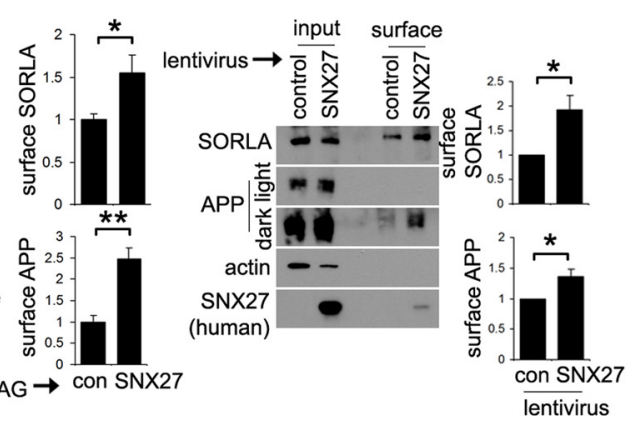

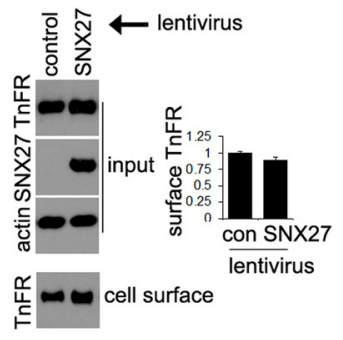
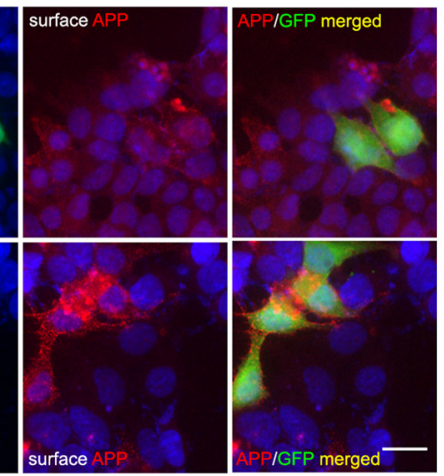

Figure 4. SNX27 overexpression can enhance SORLA and APP surface distribution. Overexpression of the SNX27 retromer component specifically promotes surface SORLA and APP distribution. $\boldsymbol{A}$, myc-tagged retromer components were coexpressed with SORLA in HEK293T cells, and surface SORLA was assayed by surface biotinylation. $\boldsymbol{B}$, myc-tagged retromer components were expressed in HEK293 cells stably expressing swAPP, and surface APP was assayed by surface biotinylation. C, HEKswAPP cells were transfected with control or FLAG-SNX27 vectors and analyzed for cell surface protein levels $72 \mathrm{~h}$ after transfection by surface biotinylation. Primary cortical neurons were transduced with control or lentiviruses expressing human SNX27 for $4 \mathrm{~d}$ at DIV17, in which neurons were biotin surface labeled and cell surface streptavidin precipitates were analyzed by immunoblotting. Graphs represent surface SORLA, APP, or TfnR normalized to total cellular levels (mean \pm SE from a minimum of 3 experiments), ${ }^{*} p<0.05,{ }^{* *} p<0.003$. D. HEKswAPP cells were transfected with control or SNX27-expressing plasmid vectors (with GFP expression from a downstream IRES element) and surface labeled for APP using B436 as described above.

receptor (TfnR) distribution (Fig. 3C, right panels). Using an APP antibody targeting the extracellular surface proximal to the $\mathrm{A} \beta$ transmembrane region (B436), we found that APP surface labeling in HEKswAPP cells observed under steady-state conditions were reduced with SNX27 siRNA treatment (Fig. 3D). These results demonstrate that reductions in SNX27 levels can also attenuate cell surface SORLA and APP distribution.

We also determined whether SNX27 overexpression could enhance surface APP and SORLA distribution. We found that overexpression of SNX27 could enhance both surface SORLA (Fig. $4 A, C$, left panels) and surface APP (Fig. 4B, C, left panels) levels in HEKswAPP cells, in which relatively little or no effect was observed with overexpression of other retromer components (Fig. 4A,B). Furthermore, SNX27 overexpression was observed to enhance surface SORLA and APP distribution, with relatively little effect on surface TfnR distribution in cortical neurons transduced with SNX27 lentiviral constructs (Fig. 4C, right panels). In support of our biotin surface labeling results, we also observed an increase in cell surface APP levels by anti-APP antibody surface labeling and immunofluorescence (Fig. $4 D$ ).

Together, these results strongly indicate that SNX27 mediates cell surface distribution of SORLA and APP.

\section{SNX27 and SORLA mediate endosome-to-cell surface APP recycling}

Using a cleavable disulfide biotin cell surface labeling method described previously (Wang et al., 2013), we labeled cell surface components in HEKswAPP cells and tracked the internalization and resurfacing kinetics of SORLA and APP. Surface-labeled SORLA and APP both achieved peak internalization at $15 \mathrm{~min}$ and consequently sorted back to the cell surface within 30-60 $\min$ (Fig. 5A). We note that, although APP almost entirely recycled to the cell surface $60 \mathrm{~min}$ after internalization, much of SORLA was retained internally. This might suggest that SORLA may be trafficked to other intracellular sites such as the Golgi after internalization, whereas APP is efficiently recycled to the cell surface. We also tracked APP endosome-to-cell surface trafficking using this method and observed redistribution of internalized APP to the cell surface between 30 and 60 min (Fig. 5B).

We then determined the individual effects of siRNAmediated SNX27 and SORLA depletion on APP internalization and endosome-to-cell surface recycling kinetics. We found no significant difference in APP internalization in HEKswAPP cells transfected with control, SNX27, and SORLA siRNA (Fig. 5C). However, we observed slower endosome-tocell surface redistribution of APP in both SNX27 and SORLA siRNA-transfected cells (Fig. 5D). These results suggest that SNX27 and SORLA may both be required for efficient APP endosome-to-cell surface recycling. Because a role for SNX27mediated endosome-to-cell surface distribution has been established for numerous plasma membrane components, including GLUR1, GIRK, $\beta$ AR, and GLUT1 (Lunn et al., 2007; Temkin et al., 2011; Steinberg et al., 2013; Wang et al., 2013), 


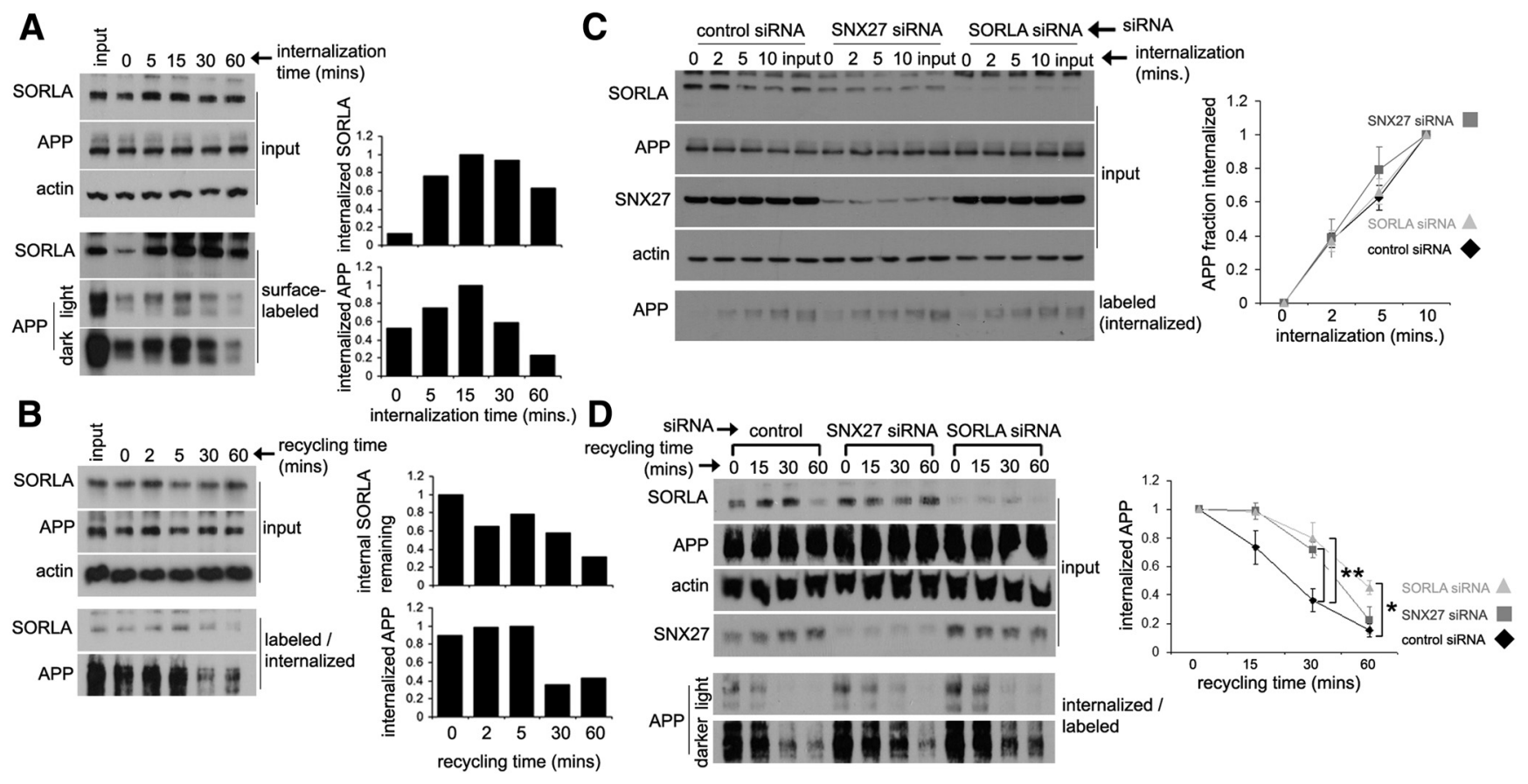

Figure 5. SNX27 and SORLA influence APP recycling kinetics after internalization. A, HEKswAPP cells were surface biotinylated with NHS-S-S-biotin, and surface labeled components were allowed to internalize and resurface for the time indicated. Surface components were cleaved with reduced glutathione, and biotinylated (internalized components) were precipitated using streptavidin agarose. Note that SORLA and APP achieved maximal internalization between 15 and $30 \mathrm{~min}$. B, Surface biotinylated components were internalized for 20 min in HEKswAPP cells in which remaining surface label was cleaved with reduced glutathione. The biotinylated surface label was allowed to resurface for the time indicated, and recycled surface components were analyzed by immunoblotting as indicated. C, HEKswAPP cells were transfected with the siRNAs as indicated and internalized for the time indicated, whereby surface components were cleaved with reduced glutathione. A non-internalized/non-cleaved input control sample is included for the indicated treatments. D, HEKswAPP cells were transfected with the siRNAs indicated, and APP recycling to the cell surface was assayed as in $\boldsymbol{B}$. All graphs depict mean \pm SE from a minimum of three independent experiments, ${ }^{*} p<0.05,{ }^{* *} p<0.01$.

this suggests that SNX27 may also mediate endosome-to-cell surface recycling of a SORLA/APP complex.

Using sucrose gradient fractionation, we found that relatively little SORLA and APP distributed to endosome-enriched fractions under steady-state conditions in HEKswAPP cells (Fig. 6). However, SORLA and APP distribution to endosomal fractions were enhanced with SNX27 siRNA transfection, and a similar redistribution of APP to endosome-enriched fractions was observed with SORLA siRNA transfection (Fig. 6A,B). In good agreement with APP distribution as observed by fractionation, we found that intracellular APP primarily localized at nonendosomal compartments by immunofluorescence in cultured HEKswAPP cells and neurons (Fig. $7 A, B$, top panels). Rather, localization of APP in cortical neurons was observed to have greater overlap with Golgi markers such as TGN38 under steady-state conditions (Fig. 7C, bottom panels). This suggests that endosomal clearance mechanisms may normally limit APP distribution to endosomes. In support of this notion, we also found that SNX27 siRNA transfection in HEKswAPP cells resulted in an increased overlap in APP and SORLA localization with endosomal markers such as EEA1 (Fig. 7C,D).

Because efficient recycling of endosomal to the cell surface could bypass trafficking of internalized components to late endosomal/ lysosomal compartments, we also determined whether SNX27 and SORLA depletion could influence APP localization at lysosomes. Although we observed poor overlap between LAMP1 and APP in control siRNA-transfected HEKswAPP cells, we observed increased LAMP1/APP overlap in both SNX27 and SORLA siRNA-transfected cells (Fig. 8). These results further indicate that APP and SORLA are components that are essentially absent from endosomes/lysosomes, presumably because of efficient SNX27-mediated recycling to the cell surface. As such, impairment of endosome-to-cell surface clearance machinery can induce SORLA and APP accumulation in endosomes, which can enhance subsequent passage of APP to late endosomes/lysosomes.

Together, these results indicate that SNX27 and SORLA are trafficking components that can affect endosome-to-cell surface APP recycling and redistribution.

\section{SNX27 and SORLA interact to mediate non-amyloidogenic APP processing}

Our results so far indicate that SNX27 interacts with a SORLA/ APP complex and that SNX27 and SORLA can influence endosome-to-cell surface APP trafficking. Both SNX27 and SORLA have been characterized previously as neuroprotective components that are required to limit amyloidogenic processing of APP to A $\beta$. The results here suggest that SNX27 and SORLA may function cooperatively as a protective shunt mechanism to redistribute APP from the endosome to the cell surface. Because amyloidogenic APP processing at acidified endosomes and non-amyloidogenic APP cleavage at the cell surface occurs in a mutually exclusive manner, our results so far suggest that SNX27 may enhance SORLA/APP surface distribution to promote non-amyloidogenic APP processing. In support of this notion, SNX27 overexpression in HEKswAPP cells produces a striking increase in $\operatorname{sAPP} \alpha$ levels, indicating an enhancement in cell surface-dependent $\alpha$-secretase cleavage (Fig. 9A). We also observed an accumulation of CTF $\alpha$ with SNX27 overexpression, which may be produced in combination with increased $\alpha$-secretase cleavage and SNX27dependent inhibition of the $\gamma$-secretase complex described previously (Fig. 9A; Wang et al., 2014b). 


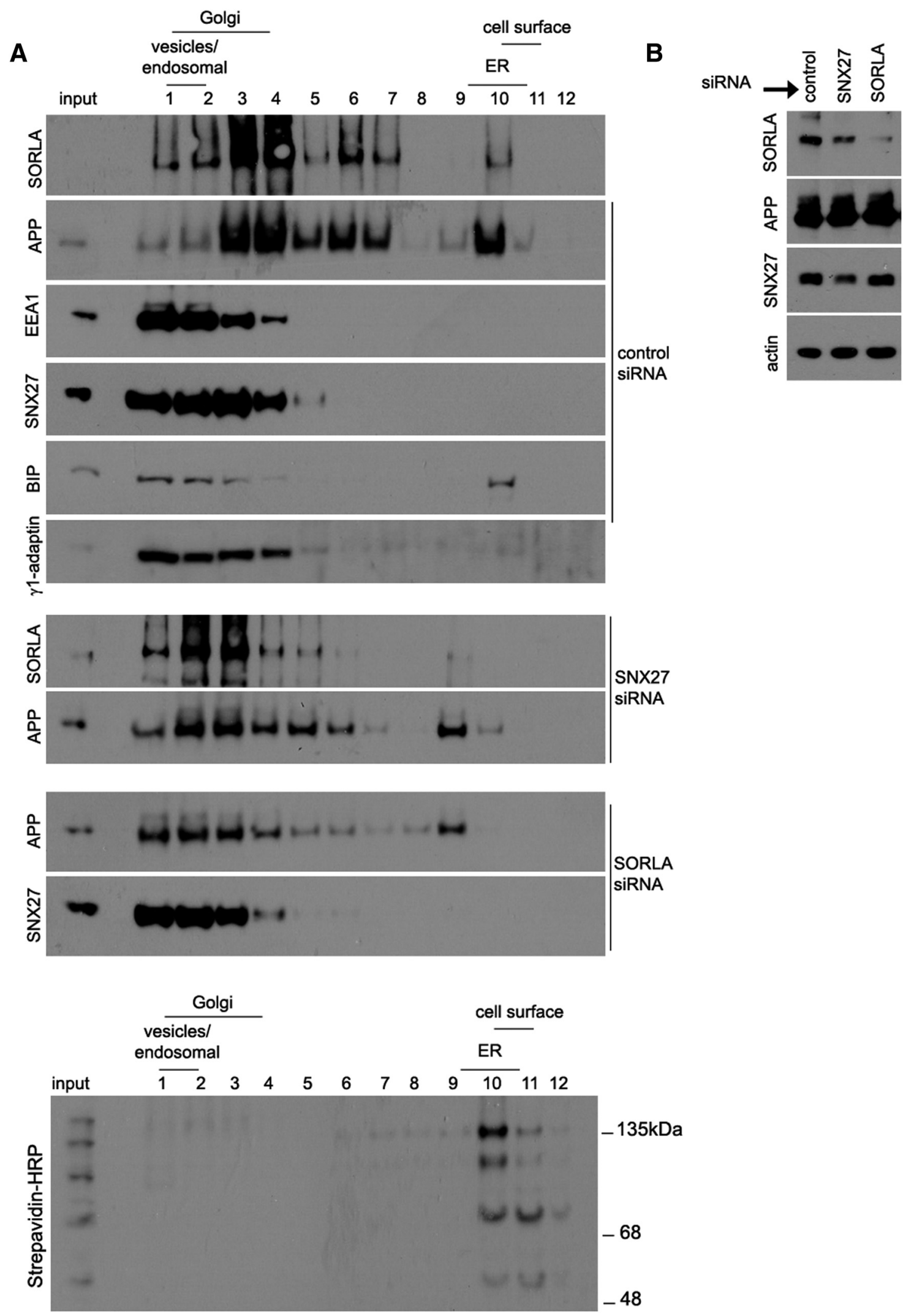

Figure 6. SNX27 and SORLA depletion can influence subcellular APP distribution. A, HEKswAPP cells were transfected with control, SNX27, or SORLA siRNAs, harvested and subjected to sucrose density fractionation as described in Materials and Methods, and immunoblotted for the components indicated. Untreated cells were surface biotinylated, and sucrose density fractions were probed with streptavidin-HRP to detect cell surface cell surface components as indicated. $\boldsymbol{B}$, Lysates from the input from $\boldsymbol{A}$ were probed for SORLA and SNX27 depletion by immunoblotting. 
A

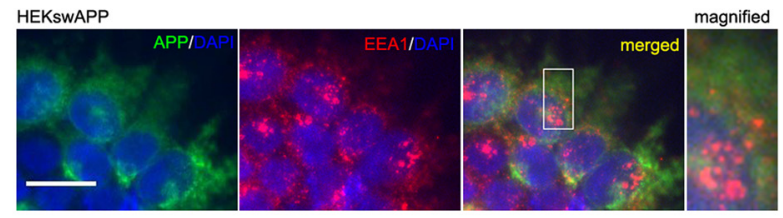

C

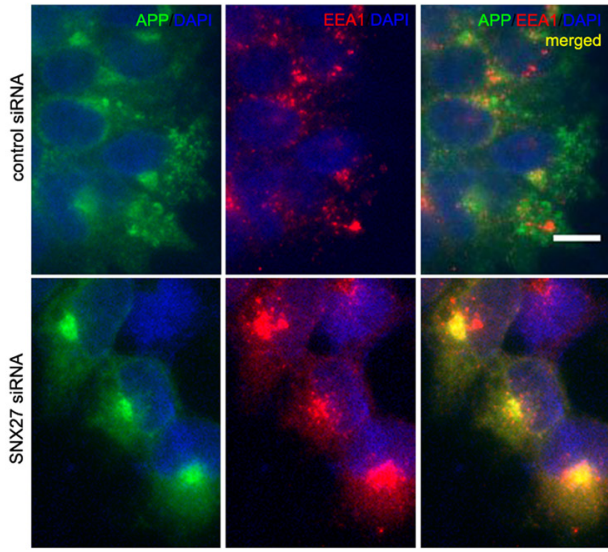

B

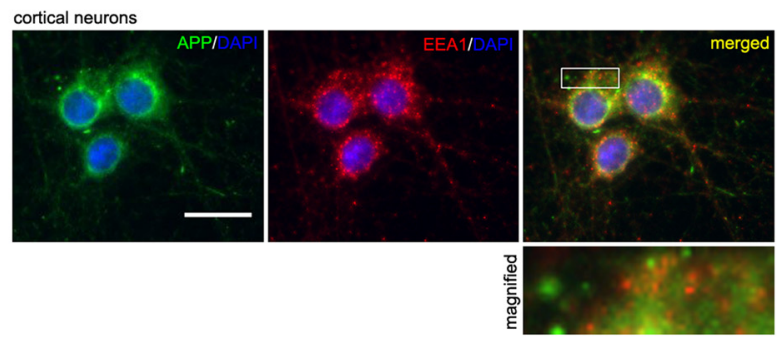

cortical neurons
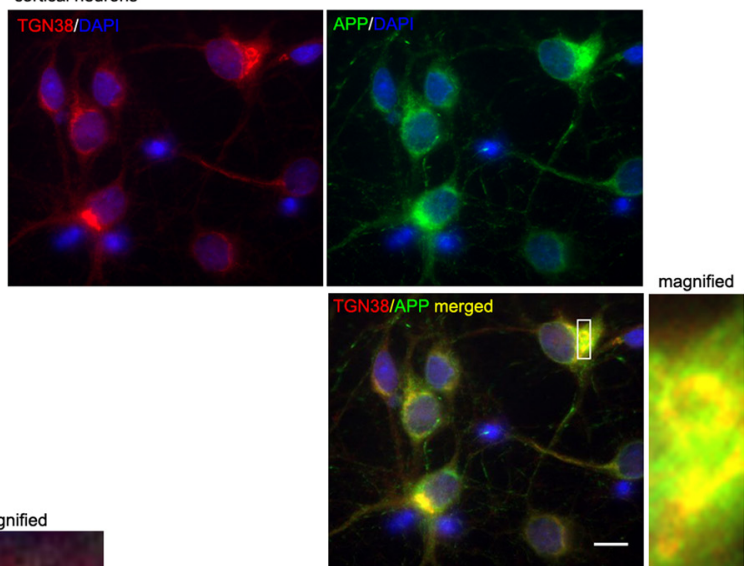

magnified
D

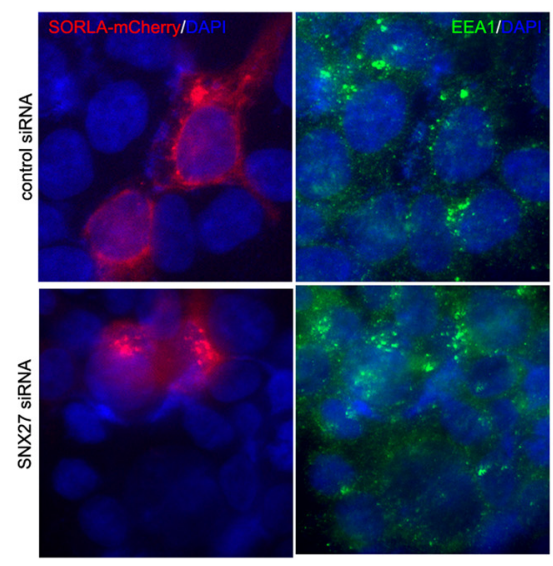

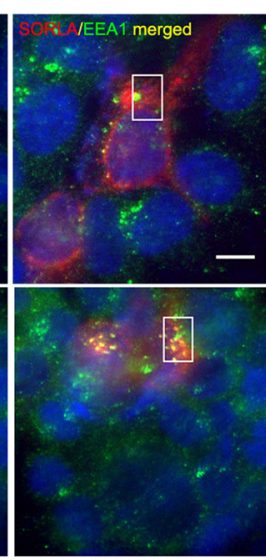

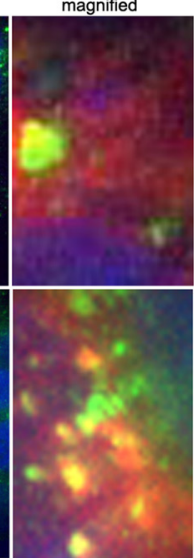

Figure 7. SNX27 depletion enhances APP and SORLA distribution to endosomal compartments. A, HEKswAPP cells were stained for APP (green) and EEA1 (red) as indicated. Note the limited colocalizing overlap between EEA1 and APP under steady-state conditions (scale bar, $10 \mu \mathrm{m}$ ). B, APP localization in cortical neurons. Cortical neurons were stained for APP (green) with EEA1 (red; top row; scale bar, $10 \mu \mathrm{m}$ ) or TGN38 (red; bottom row; scale bar, $5 \mu \mathrm{m}$ ) as indicated at DIV7. Note the limited colocalizing overlap between EEA1 and APP in neurons under steady-state conditions. C, HEKswAPP cells transfected with control or SNX27 siRNA were processed for immunocytochemistry to detect APP (green) and EEA1 (red) colocalizing overlap. D, HEKswAPP cells transfected with control or SNX27 siRNA as above were subsequently transfected with plasmid vectors expressing SORLA-mCherry; cells were fixed and stained for EEA1 to determine SORLA/endosome overlap. Scale bars: $C, D, 5 \mu \mathrm{m}$.

Given that SNX27 affects cargo distribution to numerous targets through its PDZ domain, we questioned whether attenuating SNX27/SORLA interactions through mutation of the PDZ domain could affect SORLA/APP surface distribution and consequent APP processing. To this end, we individually introduced mutations into residues conserved between SNX27, SHANK2, and NHERF2 PDZ regions within each secondary structural element in a GST-SNX27 expression construct and assayed for interactions with APP and SORLA in HEKswAPP cells (Fig. 9B). We found that SORLA/SNX27 interactions were influenced most dramatically by mutation of an L130 residue within the terminal $\beta$ strand within the PDZ domain (Fig. 9B). Integrating this mutation into a FLAG-SNX27 overexpression construct, we found that SNX27 overexpression could enhance SORLA/APP surface distribution and $\operatorname{sAPP} \alpha$ generation, whereas overexpression of the SORLA-refractory L130Q mutation had markedly reduced effects on surface SORLA/APP distribution and SAPP $\alpha$ produc- tion (Fig. 9C). This provides an additional indication that SNX27-mediated surface SORLA/APP distribution and SAPP $\alpha$ generation is dependent on SNX27/SORLA interactions.

We next determined the effects of modulating SORLA levels on APP processing. We observed a decrease in $\operatorname{sAPP} \alpha$ with SORLA overexpression and increased $\operatorname{sAPP} \alpha$ accumulation with SORLA depletion (Fig. 9D,E). Similarly, dramatic increases in $\operatorname{SAPP} \alpha$ were observed with SORLA deletion in mouse knock-out models (Rohe et al., 2008). Because our results suggest that surface SORLA/APP levels and APP cleavage may be dependent on SNX27, we assayed whether SNX27mediated elevation of sAPP $\alpha$ levels was sensitive to reductions in SORLA by siRNA transfection. We observed an attenuated SNX27-dependent increase in both CTF $\alpha$ and $\operatorname{sAPP} \alpha$ levels with SORLA siRNA transfection, indicating that SNX27dependent enhancement of non-amyloidogenic APP processing is SORLA dependent (Fig. 9F,G). This suggests that 

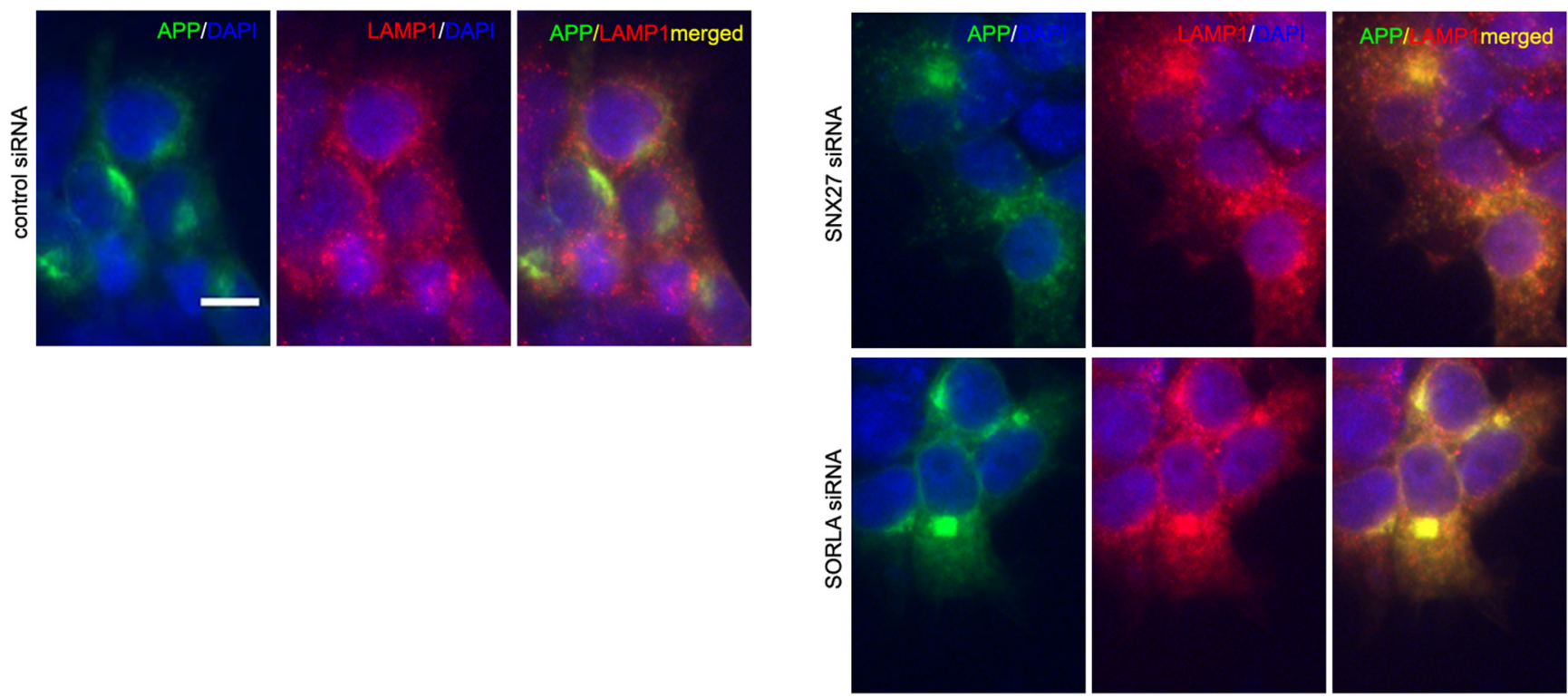

Figure 8. SNX27 or SORLA siRNA transfection promotes APP accumulation in lysosomal compartments. HEKswAPP cells were transfected for the siRNAs as indicated and costained for APP (green) and LAMP1 (red). Scale bar, $5 \mu \mathrm{m}$.

interfering with SORLA-dependent endosome-to-cell surface trafficking mechanisms may also abrogate non-amyloidogenic APP processing.

SORLA expression has been reported previously to reduce $\mathrm{A} \beta$ production in both cultured cells and mouse models (Andersen et al., 2005; Caglayan et al., 2014). Because these results so far suggest that suppression of amyloidogenic APP processing by SNX27 and SORLA may be interdependent, we determined whether reductions in $A \beta$ production through SORLA overexpression could be influenced by SNX27 reduction. As expected, we observed reductions in $\mathrm{A} \beta$ production with SORLA overexpression and elevated $\mathrm{A} \beta$ levels with SNX27 siRNA transfection (Fig. $9 H$ ). Strikingly we found that SORLA overexpression no longer attenuated $\mathrm{A} \beta$ production when SNX27 was downregulated, strongly indicating that non-amyloidogenic APP processing by SORLA is primarily dependent on SNX27. Together, these results suggest that SNX27/SORLA interactions can form a shunt mechanism to promote surface APP distribution and nonamyloidogenic processing.

\section{SNX27 facilitates SORLA and APP distribution to PSD-enriched membranes}

Having established that SNX27 mediates SORLA and APP distribution to the cell surface and because SORLA-mediated reductions in $\mathrm{A} \beta$ production are dependent on SNX27 in HEKswAPP cell lines (Fig. 9H), we determined whether SNX27 had similar effects on SORLA/APP membrane distribution in TG2576 mouse lines expressing the human Swedish APP variant. To this end, we combined TG2576, SORLA-Rosa26, and SNX27 deletion alleles through consecutive mating of TG2576 in combination with SORLA-Rosa26 and $S N X 27^{+/-}$animals and screened for TG2576 SNX27 WT and SNX27 $7^{+-}$progeny in SORLA-Rosa26 WT and TG overexpression backgrounds. TG2576 mice were killed at 8 weeks of age, and hippocampal tissue was processed for membrane/PSD enrichment and assayed for APP/SORLA distribution by immunoblotting.

We observed that SORLA and APP appeared in cytosolic and membrane fractions and were relatively less abundant in PSDenriched fractions (Fig. 10A). Our fractionation protocol parti- tioned presynaptic markers such as synaptophysin (SVP38) to Triton X-100-soluble (TX-sol) membrane fractions and PSD markers such as PSD95 to Triton X-100-insoluble PSDenrichment fractions (Fig. 10A). Although we observe mild elevations in SORLA in total lysates from our mixed SORLARosa26 background ( $<50 \%)$, we find that SORLA enrichment is nearly threefold in PSD fractions (Fig. 10B,C). We find that SNX27 haploinsufficiency in SORLA-Rosa26 TG mouse lines had a significant effect on reducing SORLA and APP levels in PSD-enriched fractions, in which SNX27 $7^{+/-}$had less of an effect in reducing SORLA and APP in TX-sol membrane fractions (Fig. $10 B, C)$. Because our results in cells and dissociated neurons indicate that SNX27 can enhance SORLA and APP distribution to the cell surface, these results give an additional indication that SNX27 can mediate distribution of SORLA and APP to PSDassociated membranes in vivo.

\section{Discussion}

The distribution of the amyloid precursor component APP to the cell surface or endosomal compartments is critical in determining its eventual processing through non-amyloidgenic or amyoidogenic pathways. Because proteolytic cleavage through these pathways occur in a mutually exclusive manner, enhanced distribution in favor of either pathway may ultimately influence proteotoxic $\mathrm{A} \beta$ generation and eventual $\mathrm{AD}$ pathophysiology (Fig. $10 D)$. Here, we describe physical and functional interactions between the APP trafficking component SORLA and SNX27, which has essential functions in diverting internalized transmembrane endosomal components back to the cell surface (Fig. 10D). Although previous evidence has indicated that SORLA has a predominant role in trafficking APP to the Golgi (Andersen et al., 2005; Rogaeva et al., 2007; Fjorback et al., 2012), whether SORLA could also mediate endosome-to-cell surface APP recycling has been essentially unexplored.

Proteomic analysis of cell surface components reduced with SNX27 siRNA downregulation previously implicated APP as a potential SNX27 trafficking target (Steinberg et al., 2013). However, failure to detect APP in the SNX27 interactome might suggest that a bridging component may be required to direct APP 
A

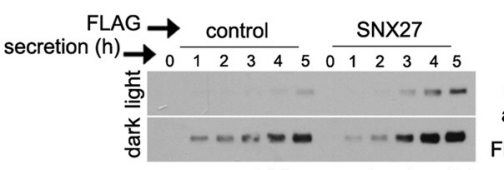

$$
\begin{aligned}
& \text { SAPP } \alpha \text { secretion (media) SAPP } \alpha \\
& \text { SAPP } \alpha \cdots \text { secreted }
\end{aligned}
$$$$
\begin{array}{ll}
\mathrm{A} A \mathrm{C} \\
\mathrm{APP} \alpha
\end{array}
$$
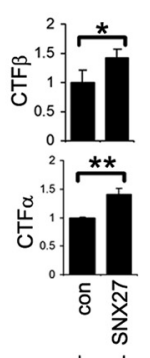

con SNX27

D
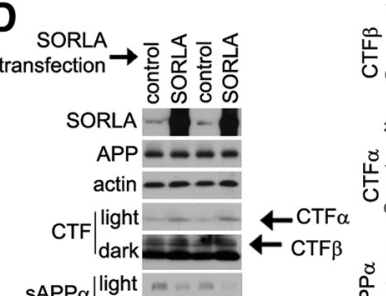

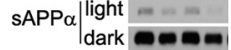

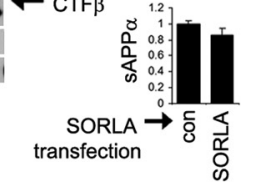

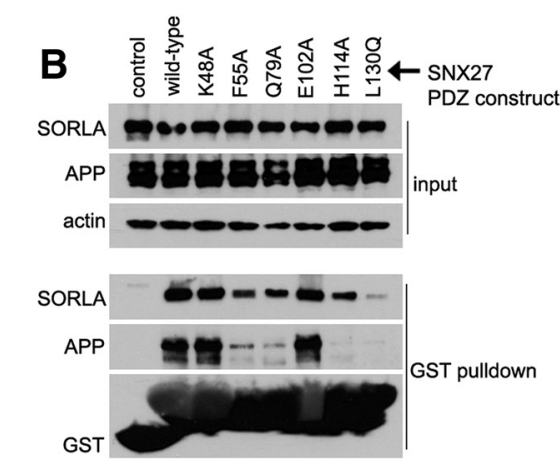

F $\quad$ FLAG $\rightarrow$ con SNX27 con SNX27
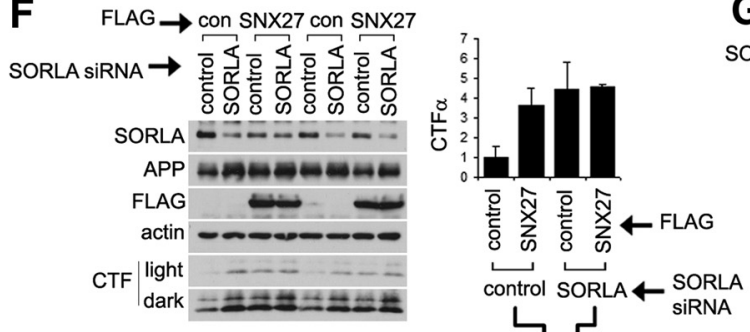

G FLAG $\rightarrow$ con SNX27 con SNX27 SORLA siRnA $\rightarrow$ 은

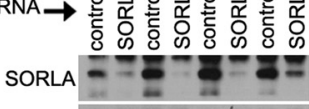

E
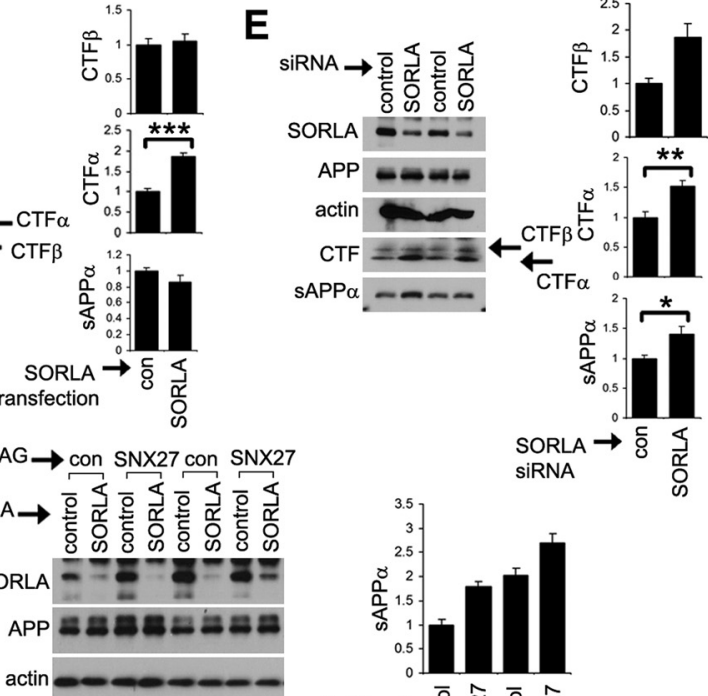

FLAG
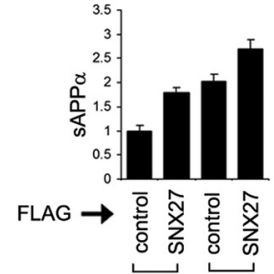

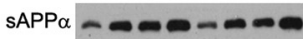

SORLA $\rightarrow$ control SORLA siRNA
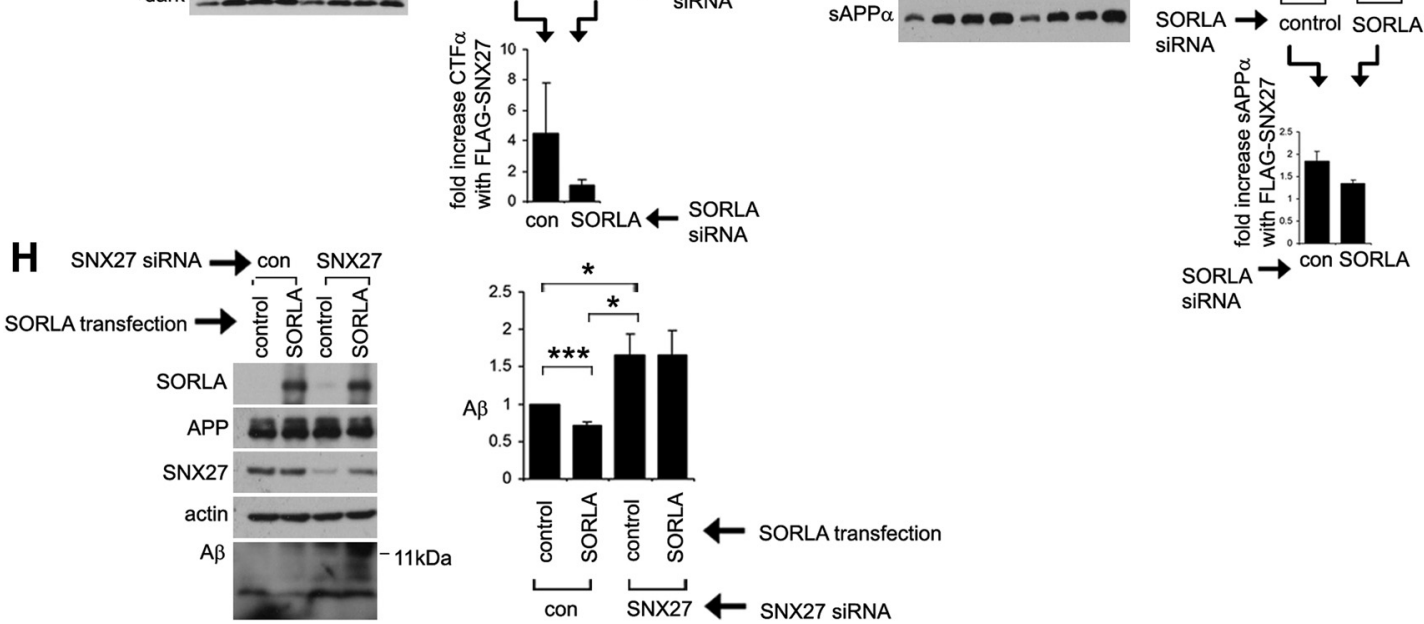

SiRNA

Figure 9. SNX27 and SORLA interdependently promote non-amyloidogenic APP cleavage.A,HEKswAPP cells transfected with control or FLAG-SNX27 vectors were allowed to secrete soluble APP species for the time indicated, and SAPP $\alpha$ was detected by immunoblotting with the B436 antibody. Lysates were also generated, and APP or CTFs were quantified by immunoblotting as indicated. B, GST-SNX27 PDZ constructs were transfected and expressed in HEKswAPP cells and precipitated with glutathione Sepharose. SORLA and APP coprecipitation was visualized by immunoblotting; L130Q PDZ constructs demonstrated marked attenuation in SORLA/APP coprecipitation. C, Empty vector, FLAG-SNX27, or L130Q constructs were transfected in HEKswAPP cells, and media were conditioned for sAPP $\alpha$ secretion as in $A$ or labeled for surface components. Surface SORLA/APP levels were precipitated with streptavidin agarose and visualized by immunoblotting; quantified surface SORLA/APP levels normalized against inputs are shown in the adjacent graphs. D, HEKswAPP cells were transfected with control or SORLA-overexpression vectors and immunoblotted for the components indicated. SAPP $\alpha$ was also detected in conditioned media from transfected cells. E, HEKswAPP cells transfected with control or SORLA siRNA were immunoblotted for the components indicated, in which media conditioned for $5 \mathrm{~h}$ was collected for sAPP $\alpha$ immunoblotting. F, HEKswAPP cells were transfected with control or SORLA siRNAs as indicated and retransfected with control or FLAG-SNX27 vectors as indicated $24 \mathrm{~h}$ later. Cells were incubated for an additional $48 \mathrm{~h}$ and immunoblotted for the components as indicated. To quantify CTF $\alpha$ cleavage, band intensity for the four treatments were quantified, and the fold CTF $\alpha$ increase with SNX27 overexpression was compared between control and SORLA siRNA transfections as indicated. G, HEKswAPP cells were transfected with control or SORLA siRNA oligos and contro//FLAG-SNX27 vectors as in $\boldsymbol{F}$, and conditioned media was analyzed for sAPP $\alpha$ secretion by immunoblot. SAPP $\alpha$ intensity was normalized relative to control siRNA/vector control transfected cells, and relative fold increases with $\mathrm{SNX} 27$ overexpression were compared between control and SORLA siRNA samples. $\boldsymbol{H}$, Control or SNX27 siRNA-transfected cells were retransfected with control or SORLA overexpression vectors, and conditioned media was TCA precipitated and immunoblotted for A $\beta$. All graphs depict mean \pm SE from at least three experiments, ${ }^{*} p<0.05,{ }^{* *} p<0.01,{ }^{* * *} p<0.005$. 

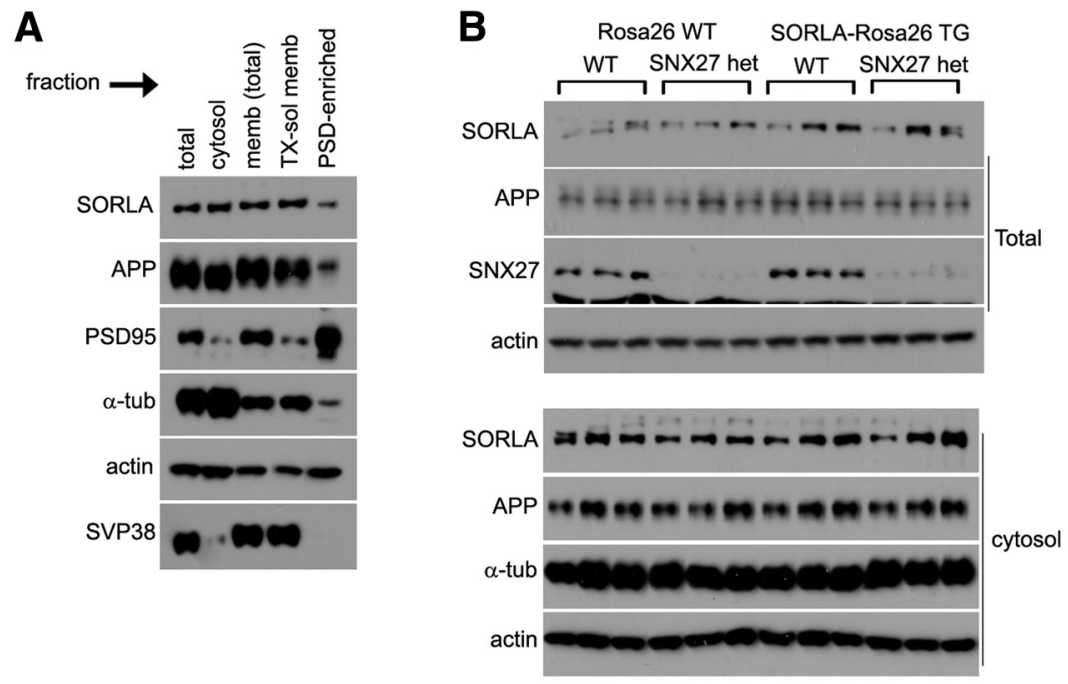

C
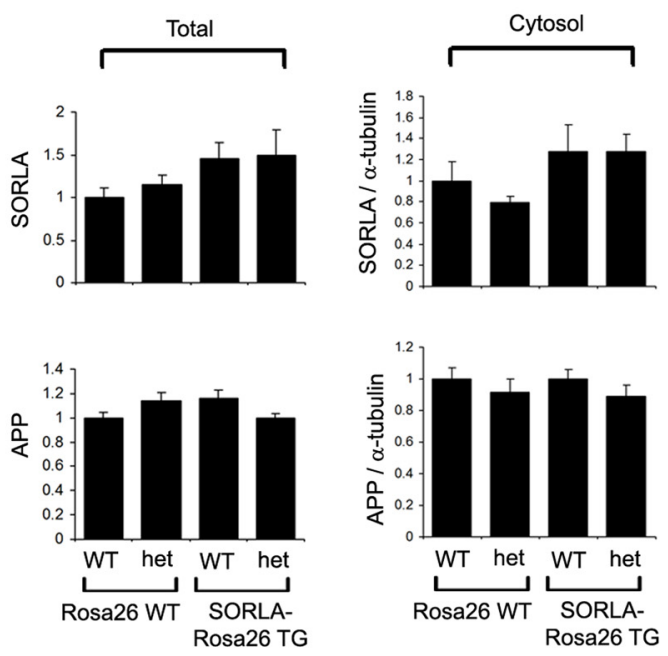
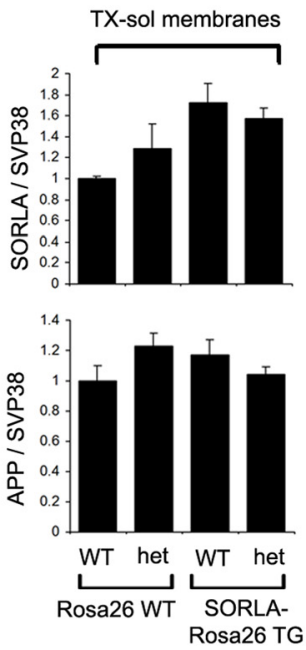
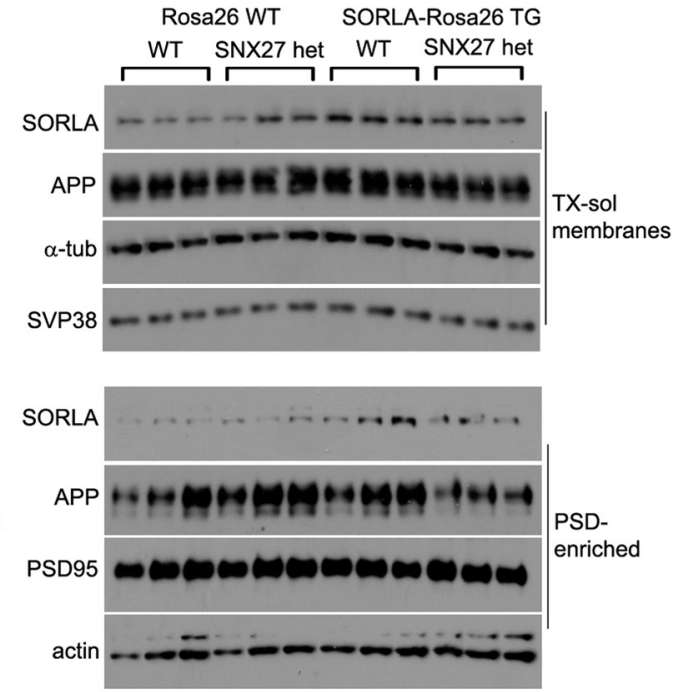

PSD-enriched

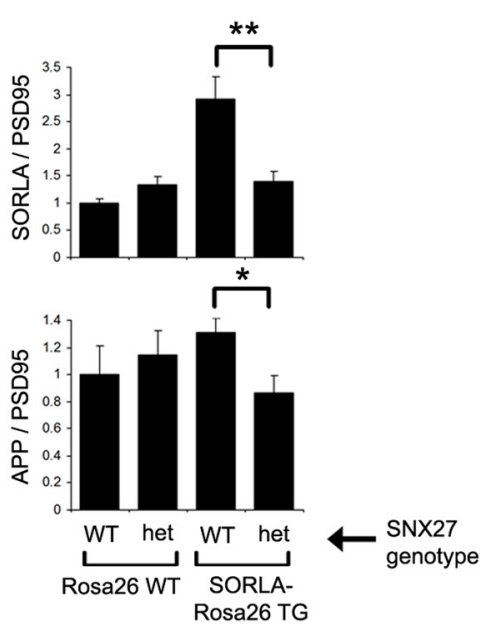

D
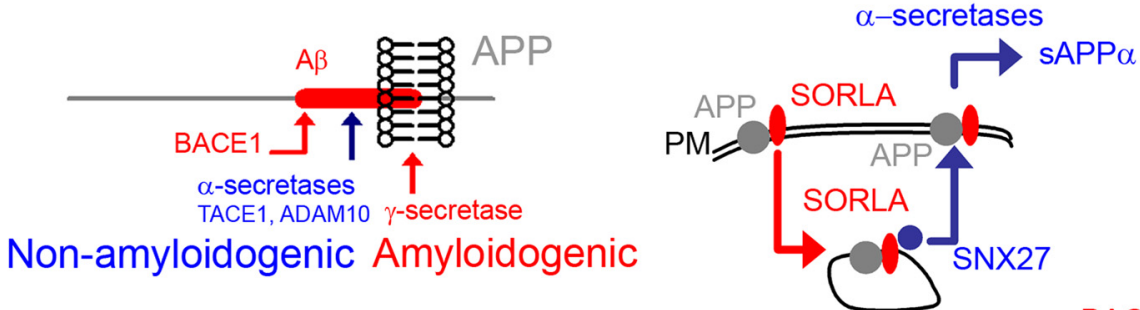

Amyloidogenic

early endosome

BACE1 cleavage

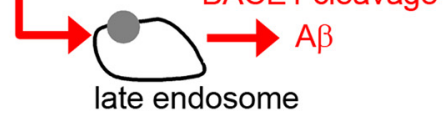

Figure 10. Model for enhanced non-amyloidogenic APP recycling to the cell surface through SNX27/SORLA interactions. A-C, SNX27 haploinsufficiency affects SORLA/APP distribution to hippocampal PSD membranes in vivo in a SORLA-Rosa26 background. $A$, TG2576 mouse hippocampus was homogenized and subjected to membrane fractionation as described in Materials and Methods. Equal protein quantities were loaded for each fraction, and components were immunoblotted as indicated. $\boldsymbol{B}$, Hippocampal tissue from TG2576 mice were dissected, homogenized, and subjected to fractionation as in $\boldsymbol{A}$. Total, cytosolic, TX-sol, and PSD-enriched fractions were generated and immunoblotted for various components as indicated. C, Hippocampal tissue from six mice of each genotype were immunoblotted for SORLA and APP from various fractions, in which SORLA and APP were subjected to densitometric quantification. SORLA/APP from cytosolic fractions were normalized to $\alpha$-tubulin, whereas TX-sol and PSD-enriched fractions were normalized to SVP38 (synaptophysin) and PSD95, respectively. All values were normalized to averages from SNX27/ SORLA-Rosa26 WT values set to 1.0. Significance values were determined by nonpaired Student's t tests $\left({ }^{*} p<0.03,{ }^{* *} p<0.01\right.$ ). D, Model for enhanced non-amyloidogenic APP recycling to the cell surface through SNX27/SORLA interactions. Left, Amyloidogenic liberation of the A $\beta$ region (red) is derived from full-length APP through sequential cleavage by BACE1 and the $\gamma$-secretase complex; non-amyloidogenic cleavage by $\alpha$-secretases (blue) at the cell surface precludes A $\beta$ generation. Right, Model for an SNX27/SORLA shunt to limit amyloidogenic A $\beta$ generation. SNX27 interactions with the SORLA cytosolic tail at early endosomes can enhance cell surface APP recycling, thereby enhancing cell surface cleavage products such as sAPP $\alpha / C$ TF $\alpha$ (blue) and attenuating amyloidogenic cleavage of $\mathrm{A} \beta$ (red). 
trafficking in vivo (Steinberg et al., 2013). Our results implicate SORLA as a likely candidate by which SNX27 diverts APP from the endosome to the cell surface (Fig. 10D). Although both SORLA and SNX27 have been characterized previously as trafficking components that reduce the amyloidogenic production of $\mathrm{A} \beta$, whether these two components worked interdependently to attenuate $A \beta$ levels was unknown. Importantly, our results show that modulation of these two components can coordinately influence non-amyloidogenic $\operatorname{sAPP} \alpha$ production and reductions in $\mathrm{A} \beta$ generation. However, we note that, under steady-state conditions, APP is primarily absent from cell surface and endosome compartments and may therefore be predominantly affected by Golgi sorting signals. In agreement with this, our results indicate that modulation of SNX27 levels has only modest effects in influencing $\mathrm{A} \beta$ generation (Wang et al., 2014b), suggesting that endosomal trafficking pathways normally compete with APP Golgi retention/retromer recycling pathways under steady-state conditions.

SORLA has been reported to reduce $\mathrm{A} \beta$ accumulation through several mechanisms, such as diverting APP traffic from the endosome to the Golgi (Fjorback et al., 2012), inhibiting BACE1/APP interactions (Spoelgen et al., 2006), inhibiting APP oligomerization (Schmidt et al., 2012), and diverting A $\beta$ traffic to lysosomes (Caglayan et al., 2014). Interestingly, SNX27 may similarly have a pluralistic role in limiting $\mathrm{A} \beta$ generation in part through inhibiting the $\gamma$-secretase complex as reported previously (Wang et al., 2014b) and in part through SORLA/APP trafficking mechanisms described here. A $\beta$ reductions with SNX27 overexpression is typically modest $(\sim 10-20 \%$; Wang et al., $2014 \mathrm{~b}$ ), and we also observed SNX27-mediated reduction in A $\beta$ levels with SORLA siRNA transfection $(\sim 15 \%$; our unpublished results). Together, this suggests that SNX27-dependent A $\beta$ reduction can occur through the inhibition of $\gamma$-secretase in the absence of SORLA and that its ability to limit A $\beta$ generation is not confined to its SORLA/APP trafficking function. Furthermore, we also observe that perturbations in SNX27 or SORLA in our experiments fail to mediate any dramatic changes in APP levels in HEKswAPP cells and in primary neurons. This indicates that, although we describe changes in $\alpha, \beta$, and $\gamma$-secretasedependent APP cleavage products with SNX27 and SORLA perturbations, both cells and primary neurons maintain a relatively stable APP pool.

Synaptic loss has been linked to cognitive decline in $\mathrm{AD}$, and it has been postulated that oligomeric $\mathrm{A} \beta$ species is a key underlying component in driving pathophysiological synaptotoxicity (Tu et al., 2014). This may suggest that site-specific A $\beta$ generation at synaptic sites may have exaggerated effects on synaptic structure and function in AD brain. SNX27 has been observed previously to be enriched at postsynaptic densities (Hussain et al., 2014), which may affect synaptic AMPAR recycling to the cell surface at synaptic sites (Wang et al., 2013; Hussain et al., 2014). We find that a portion of SORLA and APP partition to PSDenriched fractions, and SNX27 haploinsufficiency can attenuate SORLA/APP distribution to PSD-enriched preparations in SORLA-Rosa26 TG mice. Because oligomeric A $\beta$ has been correlated previously with synaptotoxicity in mouse models (Koffie et al., 2009), the possibility that SNX27 may regulate APP trafficking and processing at postsynaptic densities indicates that SNX27/SORLA-mediated APP surface redistribution may have site-specific effects at synaptic junctions.

SNX27 has been described previously to bind and function coordinately with members of the core retromer complex, particularly VPS26 (Steinberg et al., 2013). Interestingly, dysfunc- tion in members of the core retromer complex has also been described in AD. Depleted levels of VPS26 and VPS35 have been observed in AD patient brain (Small et al., 2005), and VPS26, VPS35, and SNX27 haploinsufficiency mouse models have been observed to exacerbate AD phenotypes (Muhammad et al., 2008; Wen et al., 2011; Wang et al., 2014b). Previous studies have also implicated VPS26 to bind the SORLA tail FANSHY region, in which mutation of the interaction site redistributes SORLA to non-Golgi sites, suggesting that VPS26 may also have a role in endosome to Golgi retrieval (Fjorback et al., 2012). Moreover, chemical chaperones that stabilize the retromer complex are found to redistribute APP from endosomes to attenuate amyloidogenic APP processing (Mecozzi et al., 2014). Unlike other members of the retromer complex, SNX27 is primarily involved in promoting endosome-to-cell surface recycling rather than endosome-to-Golgi shuttling mediated by the classical retromer complex (Cullen and Korswagen, 2012). At this point, it is unclear why APP shuttling has diverged two differing sorting pathways from the endosome. However, because both cell surface and Golgi sorting can divert APP from accumulation and amyloidogenic cleavage at lysosomes, both sorting pathways will be of future interest in developing anti-amyloidogenic drug targeting strategies.

Among genetic $\mathrm{AD}$ risk factors identified so far, a large number of risk components such as SORLA are found to vary frequently in the general population but demonstrate low penetrance in triggering $\mathrm{AD}$ onset. In addition to SORLA, a significant proportion of these frequently-varied/low-risk components comprise endocytic and intracellular trafficking components, including PICALM, BIN1, and CD2AP (Harold et al., 2009; Hollingworth et al., 2011). Together with the observation that retromer trafficking component deficiency can also aggravate $\mathrm{AD}$ phenotypes, this suggests that, although intracellular trafficking may not be a primary driver in $\mathrm{AD}$ onset, dysfunction of cellular trafficking mechanisms may alter the intracellular distribution of APP or its associated processing components in favor of amyloidogenesis. Because directly altering or inhibiting amyloidogenic processing components such as $\gamma$-secretase have failed to be clinically effective in treating $\mathrm{AD}$, targeting $\mathrm{AD}$ associated trafficking components such as SORLA/SNX27 may prove to be a good alternative in ameliorating $\mathrm{AD}$ outcome.

The involvement of SNX27 in $\mathrm{AD}$ pathogenesis is particularly interesting; although its abundance was not altered in AD, SNX27 was previously observed to disrupt and attenuate $\gamma$-secretase activity (Wang et al., 2013, 2014b). Furthermore, SNX27 levels were observed previously to be reduced in Down's syndrome through a C/EBP-dependent mechanism to induce AMPA receptor trafficking dysfunction and synaptic impairment (Wang et al., 2013). Because the repertoire of SNX27 targets is immense with a diverse subset of membrane proteins now to include SORLA, it is not surprising that SNX27 function is essential to proper physiological neuronal function.

SNX27 belongs to a large family of Sorting Nexin family of PX domain components, of which there are at least 33 members (Cullen, 2008). Because these components are defined by PX domains that primarily binds phosphatidylinositol-3,4,5-triphosphate $\left(\mathrm{PIP}_{3}\right)$, a large number of these components may intuitively have some functional role within endosomal compartments. Given that endosomes are aberrantly enlarged in $\mathrm{AD}$ and endosome-enriched $\mathrm{PIP}_{3}$ levels are reduced in human $\mathrm{AD}$ and $\mathrm{AD}$ mouse models (Cataldo et al., 2000; Nixon, 2005; Morel et al., 2013), it will also be interesting whether any of the other Sorting Nexin family members are involved in trafficking amyloidogenic components from the endosome and whether this activity involves SNX27 and SORLA. 
In summary, we present evidence for an endosomal sorting system for APP redistribution from endosomes to the cell surface involving SNX27 and SORLA trafficking components.

\section{References}

Andersen OM, Reiche J, Schmidt V, Gotthardt M, Spoelgen R, Behlke J, von Arnim CA, Breiderhoff T, Jansen P, Wu X, Bales KR, Cappai R, Masters CL, Gliemann J, Mufson EJ, Hyman BT, Paul SM, Nykjaer A, Willnow TE (2005) Neuronal sorting protein-related receptor sorLA/LR11 regulates processing of the amyloid precursor protein. Proc Natl Acad Sci U S A 102:13461-13466. CrossRef Medline

Caglayan S, Takagi-Niidome S, Liao F, Carlo AS, Schmidt V, Burgert T, Kitago Y, Fuchtbauer EM, Fuchtbauer A, Holtzman DM, Takagi J, Willnow TE (2014) Lysosomal sorting of amyloid-beta by the SORLA receptor is impaired by a familial Alzheimer's disease mutation. Sci Transl Med 6:223ra220. CrossRef Medline

Cataldo AM, Peterhoff CM, Troncoso JC, Gomez-Isla T, Hyman BT, Nixon RA (2000) Endocytic pathway abnormalities precede amyloid beta deposition in sporadic Alzheimer's disease and Down syndrome: differential effects of APOE genotype and presenilin mutations. Am J Pathol 157:277-286. CrossRef Medline

Cullen PJ (2008) Endosomal sorting and signalling: an emerging role for sorting nexins. Nat Rev Mol Cell Biol 9:574-582. CrossRef Medline

Cullen PJ, Korswagen HC (2012) Sorting nexins provide diversity for retromer-dependent trafficking events. Nat Cell Biol 14:29-37. CrossRef Medline

Eggert S, Midthune B, Cottrell B, Koo EH (2009) Induced dimerization of the amyloid precursor protein leads to decreased amyloid-beta protein production. J Biol Chem 284:28943-28952. CrossRef Medline

Fjorback AW, Seaman M, Gustafsen C, Mehmedbasic A, Gokool S, Wu C, Militz D, Schmidt V, Madsen P, Nyengaard JR, Willnow TE, Christensen EI, Mobley WB, Nykjær A, Andersen OM (2012) Retromer binds the FANSHY sorting motif in SorLA to regulate amyloid precursor protein sorting and processing. J Neurosci 32:1467-1480. CrossRef Medline

Harold D, Abraham R, Hollingworth P, Sims R, Gerrish A, Hamshere ML, Pahwa JS, Moskvina V, Dowzell K, Williams A, Jones N, Thomas C, Stretton A, Morgan AR, Lovestone S, Powell J, Proitsi P, Lupton MK, Brayne C, Rubinsztein DC, et al. (2009) Genome-wide association study identifies variants at CLU and PICALM associated with Alzheimer's disease. Nat Gen 41:1088-1093. CrossRef Medline

Herskowitz JH, Offe K, Deshpande A, Kahn RA, Levey AI, Lah JJ (2012) GGA1-mediated endocytic traffic of LR11/SorLA alters APP intracellular distribution and amyloid-beta production. Mol Biol Cell 23:2645-2657. CrossRef Medline

Hollingworth P, Harold D, Sims R, Gerrish A, Lambert JC, Carrasquillo MM, Abraham R, Hamshere ML, Pahwa JS, Moskvina V, Dowzell K, Jones N, Stretton A, Thomas C, Richards A, Ivanov D, Widdowson C, Chapman J, Lovestone S, Powell J, et al. (2011) Common variants at ABCA7, MS4A6A/MS4A4E, EPHA1, CD33 and CD2AP are associated with Alzheimer's disease. Nat Genet 43:429-435. CrossRef Medline

Huang TY, Michael S, Xu T, Sarkeshik A, Moresco JJ, Yates JR 3rd, Masliah E, Bokoch GM, DerMardirossian C (2013) A novel Racl GAP splice variant relays poly-Ub accumulation signals to mediate Racl inactivation. Mol Biol Cell 24:194-209. CrossRef Medline

Hussain NK, Diering GH, Sole J, Anggono V, Huganir RL (2014) Sorting Nexin 27 regulates basal and activity-dependent trafficking of AMPARs. Proc Natl Acad Sci U S A 111:11840-11845. CrossRef Medline

Jiang S, Li Y, Zhang X, Bu G, Xu H, Zhang YW (2014) Trafficking regulation of proteins in Alzheimer's disease. Mol Neurodegener 9:6. CrossRef Medline

Koffie RM, Meyer-Luehmann M, Hashimoto T, Adams KW, Mielke ML, Garcia-Alloza M, Micheva KD, Smith SJ, Kim ML, Lee VM, Hyman BT, Spires-Jones TL (2009) Oligomeric amyloid beta associates with postsynaptic densities and correlates with excitatory synapse loss near senile plaques. Proc Natl Acad Sci U S A 106:4012-4017. CrossRef Medline

Lauffer BE, Melero C, Temkin P, Lei C, Hong W, Kortemme T, von Zastrow M (2010) SNX27 mediates PDZ-directed sorting from endosomes to the plasma membrane. J Cell Biol 190:565-574. CrossRef Medline

Lunn ML, Nassirpour R, Arrabit C, Tan J, McLeod I, Arias CM, Sawchenko PE, Yates JR 3rd, Slesinger PA (2007) A unique sorting nexin regulates trafficking of potassium channels via a PDZ domain interaction. Nat Neurosci 10:1249-1259. CrossRef Medline

Mecozzi VJ, Berman DE, Simoes S, Vetanovetz C, Awal MR, Patel VM, Schneider RT, Petsko GA, Ringe D, Small SA (2014) Pharmacological chaperones stabilize retromer to limit APP processing. Nat Chem Biol 10: 443-449. CrossRef Medline

Morel E, Chamoun Z, Lasiecka ZM, Chan RB, Williamson RL, Vetanovetz C, Dall'Armi C, Simoes S, Point Du Jour KS, McCabe BD, Small SA, Di Paolo G (2013) Phosphatidylinositol-3-phosphate regulates sorting and processing of amyloid precursor protein through the endosomal system. Nat Commun 4:2250. CrossRef Medline

Muhammad A, Flores I, Zhang H, Yu R, Staniszewski A, Planel E, Herman M, Ho L, Kreber R, Honig LS, Ganetzky B, Duff K, Arancio O, Small SA (2008) Retromer deficiency observed in Alzheimer's disease causes hippocampal dysfunction, neurodegeneration, and Abeta accumulation. Proc Natl Acad Sci U S A 105:7327-7332. CrossRef Medline

Nixon RA (2005) Endosome function and dysfunction in Alzheimer's disease and other neurodegenerative diseases. Neurobiol Aging 26:373-382. CrossRef Medline

Offe K, Dodson SE, Shoemaker JT, Fritz JJ, Gearing M, Levey AI, Lah JJ (2006) The lipoprotein receptor LR11 regulates amyloid beta production and amyloid precursor protein traffic in endosomal compartments. J Neurosci 26:1596-1603. CrossRef Medline

Pottier C, Hannequin D, Coutant S, Rovelet-Lecrux A, Wallon D, Rousseau S, Legallic S, Paquet C, Bombois S, Pariente J, Thomas-Anterion C, Michon A, Croisile B, Etcharry-Bouyx F, Berr C, Dartigues JF, Amouyel P, Dauchel H, Boutoleau-Bretonnière C, Thauvin C, et al. (2012) High frequency of potentially pathogenic SORL1 mutations in autosomal dominant early-onset Alzheimer disease. Mol Psychiatry 17:875-879. CrossRef Medline

Rogaeva E, Meng Y, Lee JH, Gu Y, Kawarai T, Zou F, Katayama T, Baldwin CT, Cheng R, Hasegawa H, Chen F, Shibata N, Lunetta KL, PardossiPiquard R, Bohm C, Wakutani Y, Cupples LA, Cuenco KT, Green RC, Pinessi L, et al. (2007) The neuronal sortilin-related receptor SORL1 is genetically associated with Alzheimer disease. Nat Genet 39:168-177. CrossRef Medline

Rohe M, Carlo AS, Breyhan H, Sporbert A, Militz D, Schmidt V, Wozny C, Harmeier A, Erdmann B, Bales KR, Wolf S, Kempermann G, Paul SM, Schmitz D, Bayer TA, Willnow TE, Andersen OM (2008) Sortilinrelated receptor with A-type repeats (SORLA) affects the amyloid precursor protein-dependent stimulation of ERK signaling and adult neurogenesis. J Biol Chem 283:14826-14834. CrossRef Medline

Scherzer CR, Offe K, Gearing M, Rees HD, Fang G, Heilman CJ, Schaller C, Bujo H, Levey AI, Lah JJ (2004) Loss of apolipoprotein E receptor LR11 in Alzheimer disease. Arch Neurol 61:1200-1205. CrossRef Medline

Schmidt V, Baum K, Lao A, Rateitschak K, Schmitz Y, Teichmann A, Wiesner B, Petersen CM, Nykjaer A, Wolf J, Wolkenhauer O, Willnow TE (2012) Quantitative modelling of amyloidogenic processing and its influence by SORLA in Alzheimer's disease. EMBO J 31:187-200. CrossRef Medline

Small SA, Kent K, Pierce A, Leung C, Kang MS, Okada H, Honig L, Vonsattel JP, Kim TW (2005) Model-guided microarray implicates the retromer complex in Alzheimer's disease. Ann Neurol 58:909-919. CrossRef Medline

Spoelgen R, von Arnim CA, Thomas AV, Peltan ID, Koker M, Deng A, Irizarry MC, Andersen OM, Willnow TE, Hyman BT (2006) Interaction of the cytosolic domains of sorLA/LR11 with the amyloid precursor protein (APP) and beta-secretase beta-site APP-cleaving enzyme. J Neurosci 26:418-428. CrossRef Medline

Steinberg F, Gallon M, Winfield M, Thomas EC, Bell AJ, Heesom KJ, Tavaré JM, Cullen PJ (2013) A global analysis of SNX27-retromer assembly and cargo specificity reveals a function in glucose and metal ion transport. Nat Cell Biol 15:461-471. CrossRef Medline

Temkin P, Lauffer B, Jäger S, Cimermancic P, Krogan NJ, von Zastrow M (2011) SNX27 mediates retromer tubule entry and endosome-to-plasma membrane trafficking of signalling receptors. Nat Cell Biol 13:715-721. CrossRef Medline

Thinakaran G, Koo EH (2008) Amyloid precursor protein trafficking, processing, and function. J Biol Chem 283:29615-29619. CrossRef Medline

Tu S, Okamoto S, Lipton SA, Xu H (2014) Oligomeric Abeta-induced 
synaptic dysfunction in Alzheimer's disease. Mol Neurodegener 9:48. CrossRef Medline

Wang X, Zhao Y, Zhang X, Badie H, Zhou Y, Mu Y, Loo LS, Cai L, Thompson RC, Yang B, Chen Y, Johnson PF, Wu C, Bu G, Mobley WC, Zhang D, Gage FH, Ranscht B, Zhang YW, Lipton SA, et al. (2013) Loss of sorting nexin 27 contributes to excitatory synaptic dysfunction by modulating glutamate receptor recycling in Down's syndrome. Nat Med 19:473-480. CrossRef Medline

Wang X, Huang T, Xu H (2014a) Dysregulation of protein trafficking in neurodegeneration. Mol Neurodeg 9:31-39. CrossRef Medline

Wang X, Huang T, Zhao Y, Zheng Q, Thompson RC, Bu G, Zhang YW, Hong $\mathrm{W}, \mathrm{Xu} \mathrm{H}(2014 \mathrm{~b})$ Sorting nexin 27 regulates $\mathrm{A} \beta$ production through modulating $\gamma$-secretase activity. Cell Rep 9:1023-1033. CrossRef Medline

Wen L, Tang FL, Hong Y, Luo SW, Wang CL, He W, Shen C, Jung JU, Xiong
F, Lee DH, Zhang QG, Brann D, Kim TW, Yan R, Mei L, Xiong WC (2011) VPS35 haploinsufficiency increases Alzheimer's disease neuropathology. J Cell Biol 195:765-779. CrossRef Medline

Xu H, Sweeney D, Wang R, Thinakaran G, Lo AC, Sisodia SS, Greengard P, Gandy S (1997) Generation of Alzheimer beta-amyloid protein in the trans-Golgi network in the apparent absence of vesicle formation. Proc Natl Acad Sci U S A 94:3748-3752. CrossRef Medline

Zhang YW, Luo WJ, Wang H, Lin P, Vetrivel KS, Liao F, Li F, Wong PC, Farquhar MG, Thinakaran G, Xu H (2005) Nicastrin is critical for stability and trafficking but not association of other presenilin/ gamma-secretase components. J Biol Chem 280:17020-17026. CrossRef Medline

Zhang YW, Thompson R, Zhang H, Xu H (2011) APP processing in Alzheimer’s disease. Mol Brain 4:3. CrossRef Medline 